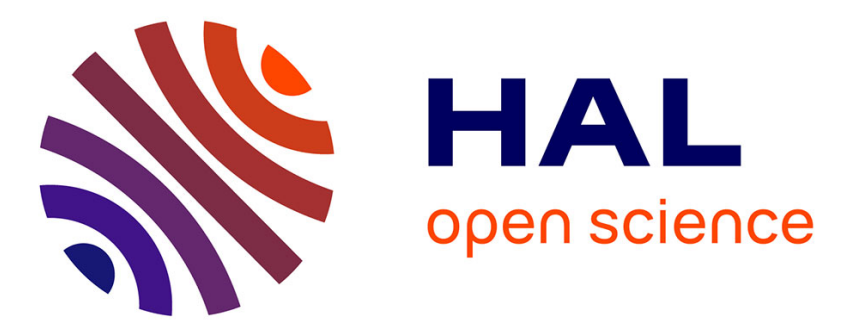

\title{
Une illustration de l'évolution des pratiques funéraires en Languedoc oriental à l'Âge du Fer : la tombe de Font de la Vie à Saint-Bauzille-de-Montmel, Hérault, Ve s. avant J.-C.
}

Bernard Dedet

\section{To cite this version:}

Bernard Dedet. Une illustration de l'évolution des pratiques funéraires en Languedoc oriental à l'Âge du Fer : la tombe de Font de la Vie à Saint-Bauzille-de-Montmel, Hérault, Ve s. avant J.-C.. Gallia Archéologie de la France antique, 1995, 52, pp.145-163. 10.3406/galia.1995.3137 . hal-01900943

\section{HAL Id: hal-01900943 \\ https://hal.science/hal-01900943}

Submitted on 16 Jan 2020

HAL is a multi-disciplinary open access archive for the deposit and dissemination of scientific research documents, whether they are published or not. The documents may come from teaching and research institutions in France or abroad, or from public or private research centers.
L'archive ouverte pluridisciplinaire HAL, est destinée au dépôt et à la diffusion de documents scientifiques de niveau recherche, publiés ou non, émanant des établissements d'enseignement et de recherche français ou étrangers, des laboratoires publics ou privés.

\section{(ㅇ)(1) $\$$}

Distributed under a Creative Commons Attribution - NonCommercial - NoDerivatives $\mid 4.0$ 


\title{
UNE ILLUSTRATION DE L'ÉVOLUTION DES PRATIQUES FUNÉRAIRES EN LANGUEDOC ORIENTAL À L'ÂGE DU FER
}

\section{La tombe de Font de la Vie à Saint-Bauzille-de-Montmel, Hérault,} $V^{e}$ s. avant J.-C.

\section{Bernard DEDET *}

\begin{abstract}
Mots clefs. Sépulture, pratiques funéraires, incinération, anthropologie, accompagnement, offrandes, Âge du Fer, Font de la Vie, Saint-
\end{abstract} Bauzille-de-Montmel, Languedoc oriental.

Key words. Crave, funerary practices, cremation, anthropology, offering, Iron Age, Font de la Vie, Saint-Bauzille-de-Montmel, Eastern Languedoc.

Résumé. La tombe de Font de la Vie est celle d'un adulte, très probablement un homme, décédé au milieu du Ve s. avant J.-C. et brûlé sur un ustrinum. Elle comprend une fosse sépulcrale, où ont été jetés en vrac, une petite partie du bûcher funèbre des os incinérés et des restes du matériel d'accompagnement fortement altérés par le feu et un dépôt annexe probablement d'offrande. Du fait de la grande indigence de la documentation en Languedoc oriental entre le débnt $d u V^{*}$ s. et celui $d u$. IT s. avant J.-C., cette décourverte constitue un jalon important permettant de discerner l'évolution des pratiques funéraires de cette région. La variété des types de traitement du corps en vigueur aux VIIIVIr s. avant J.-C. dans le complexe tumulaire disparaît au VI s. cédant la place à l'unicité : seule subsiste alors dans les derniers tumuli l'incinération avec dépôt hors de tout contenant et souvent disséminés, de quelques os brûlés accompagnés d'objets qui ne sont pas passés sur le bûcher. À partir de la fin du VTe ou du début du V s., si l'incinération reste l'exclusive dans la région, et ce durant tout le second $\hat{A} g e$ du Fer, du moins pour les individus décédés après la petite enfance, deux grands types de pratiques apparaissent : d'une part, le dépôt dans un loculus creusé en pleine terre, d'un vase-ossuaire contenant quelques os humains prélevés sur le bûcher, et des objets d'accompagnement non brûlés et complets, d'autre part, comme à Font de la Vie, le dépôt en vrac dans une petite fosse d'une partie du bîcher incluant des os incinérés et des morceaux d'un mobilier brisé sur l'ustrinum.

\begin{abstract}
The Font de la Vie grave is that of an adult, most probably a man, who died in the mid 5th century B.C. and was burnt on an ustrinum. It includes a burial pit into which were indiscriminately thrown a small part of the funeral pyre containing calcined bones and crushed remains of the pyre, as well as a smaller heap of what may have been an offering. Since the information concerning Eastern Languedoc between the early 5 th century and the early 2 nd century B.C. is rather sparse, this discover is indeed an important landmark that enables us to measure the evolution of funeral rites in this area. The variety of ways the bodies have been treated in the 8th and 7th centuries B.C. in the barrow system disappears in the 6th century, giving way to uniformity : in the later tumuli, cremation remains alone and the calcined bones lie directly in the ground, most often scattered along with a few artifacts which had not been set on the funeral pyre. From the late 6th century on, or from the early 5 th century B.C., if cremation remains exclusive in the area, and this during the whole second Iron Age, at least for people who died after infancy, two major types of rites appear : in the first place, the deposit in a loculus dug in the ground of a cist containing a few human bones taken from the pyre, as well as some intact artifacts; on the other hand, as at Font de la Vie, the dumping in a shallow pit of a small part of the pyre containing calcined bones and some other oddments on the ustrinum.
\end{abstract}

* C.N.R.S., UMR 154, 390 route de Pérols, 34970 Lattes. 
Malgré un siècle de découvertes fortuites, signalées dans la littérature archéologique, ou de recherches plus ou moins systématiques, la documentation concernant les pratiques funéraires protohistoriques du Languedoc oriental est, à l'heure actuelle, particulièrement mal répartie dans le temps comme dans l'espace. Cela est en parfaite discordance avec la représentation spatiale et temporelle de l'habitat. Deux grandes séries de tombes éclairent, en ce domaine, le début et la fin de l'Âge du Fer.

Tout d'abord, et en total contraste avec le Bronze final, où les sépulcres connus sont exceptionnels, la phase ancienne du premier Âge du Fer, antérieure à la fin du $\mathrm{VII}^{\mathrm{e}}$ s. avant J.-C., de faciès " suspendien ". Pour cette époque, ce sont plus de trois cents tumuli, édifiés en pierres en surface d'un sol rocheux, auxquels s'ajoute au moins une trentaine de dolmens plus anciens réutilisés alors à des fins funéraires. Mais ces tombes sont alors cantonnées à la partie intérieure de cette région, celle des Garrigues de l'Hérault et du Gard, tandis que les grandes plaines littorales et bas-rhodaniennes, ainsi que la côte méditerranéenne, qui présentent pourtant une densité d'habitats beaucoup plus forte et où se trouvent les agglomérations les plus étendues de tout le Languedoc oriental, pour l'époque, constituent encore de ce point de vue une véritable terra incognita. Même dans les Garrigues, on ne connaît guère que les sépultures établies sur les collines et les plateaux calcaires, et l'on ignore tout, ou presque, de celles qui furent installées dans les vallées et les dépressions aérant ce pays. Ce phénomène tumulaire, déjà attesté dans la région au moins depuis le Bronze ancien/moyen, et sans doute antérieurement au Néolithique-Chacolithique, mais à quelques exemplaires seulement, connaît un grand développement au début du premier Âge du Fer, décline très rapidement dès le commencement du VI ${ }^{\mathrm{e}} \mathrm{s}$. - seuls quatre tumuli peuvent être rapportés avec sûreté à ce siècle - et disparaît vers les environs de 500 (Dedet, 1992 et 1995).

Il faut attendre ensuite le $\mathrm{I}^{\text {er }}$ s. avant J.-C. pour retrouver, dans le Gard uniquement, une série relativement bien fournie de sépultures, une trentaine en tout, isolées, ou groupées en nécropoles d'ampleur variable et dont l'usage, parfois, continue au siècle suivant, aux abords des agglomérations principales, comme Nîmes et Beaucaire, ou secondaires, ou encore en milieu rural (Py, 1981 et 1990).

Entre ces deux ensembles, pour ces quatre siècles courant entre les derniers tumuli et les tombes du $\mathrm{I}^{\mathrm{er}} \mathbf{s}$. avant J.C., les sépultures attestées jusqu'à ces dernières années étaient extrêmement rares : quatre à la jointure des $\mathrm{V}^{e}$ et $^{\mathrm{e}} \mathrm{s}$., une à la fin du $\mathrm{IV}^{\mathrm{e}}$ ou à la première moitié du III' $s$. et cinq pour le II ${ }^{e} s$. avant J.-C. En outre, les conditions de découverte de tous ces gisements n'ont permis d'obtcnir que des données très fragmentaires sur les pratiques funéraires.

Une telle lacune dans la documentation funéraire, alors même que les habitats des $\mathrm{V}^{\mathrm{e}}$ et $\mathrm{IV}^{\mathrm{e}} \mathrm{s}$. avant J.-C. sont fort nombreux dans tout le Languedoc oriental, et ceux des III $^{\mathrm{e}}$ et II ${ }^{\mathrm{e}}$ s. avant J.-C. bien connus dans le secteur littoral et bas-rhodanien de cette région, n'a pas manqué de susciter des interrogations. Hasard des découvertes? Le temps passant, cette hypothèse devenait moins crédible. Changements dans les rites funéraires et adoption de coutumes laissant des restes plus ténus, plus fragiles, ou même ne procurant pas de trace durable? Sans doute, mais lesquels? La question vaut aussi pour la Provence où, il y a déjà une vingtaine d'années, un constat similaire était établi (Goudineau, 1975, p. 22). En 1989, la publication de sept sépultures du dernier quart du $\mathrm{III}^{\mathrm{e}}$ et de la première moitié du II ${ }^{\mathrm{e}} \mathrm{s}$. avant J.-C. au quartier du Sablas, au pied de l'oppidum contemporain d'Ambrussum (Villetelle, Hérault), en bordure du Vidourle, permettait de relancer le débat (Fiches dir., 1989, p. 27-40). Ces tombes, fort peu spectaculaires, de petites cuvettes très peu profondes avec ossements incinérés et objets d'accompagnement très incomplets et fragmentés, ont été recouvertes par les alluvions du fleuve. Leur trouvaille en 1985 est liée à la fouille de l'habitat construit au-dessus au $\mathrm{I}^{\text {er }}$ s. avant J.-C. La reconnaissance de tels vestiges suppose donc des conditions particulièrement favorables de conservation et de découverte : en d'autres lieux, de telles traces peuvent disparaître plus ou moins rapidement; masquées par une épaisse couche alluviale, elles ne peuvent être décelées par une prospection de surface; peu remarquables enfin, elles n'attirent guère l'attention en cas de mise au jour fortuite.

C'est dans un tel contexte documentaire que prennent place la découverte fortuite et la fouille, en 1993, dans les Garrigues du nord-est de l'Hérault, près du village de Saint-Bauzille-de-Montmel, au lieu-dit Font de la $V i e$, des restes d'une tombe enfouie vers le milieu du $V^{e} s$. avant J.-C. Bien qu'endommagée et sans doute incomplète, cette sépulture apporte de nombreux renseignements sur la personnalité du défunt et les pratiques funéraires mises en œuvre. Étant donné le caractère très partiel des observations faites ailleurs sur les très rares tombes de la région datées entre la fin du Vre et celle du $\mathrm{III}^{\mathrm{e}} \mathrm{s}$. avant 
J.-C., la contribution de cette découverte est particulièrement importante : elle permet d'illustrer et de mieux situer dans le temps une profonde transformation des usages funéraires protohistoriques de cette partie du Languedoc. La publication de cette sépulture présente donc deux volets. L'étude des vestiges de la tombe, du mobilier, du défunt et des pratiques dont tous ces restes témoignent, fait l'objet de la première partie. Replacées dans leur contexte du Languedoc oriental protohistorique, ces données nous permettent ensuite, dans une seconde partie, de tenter de tracer une première esquisse des grandes lignes de l'évolution de ces pratiques au milieu de l'Âge du Fer, telles qu'elles peuvent se dégager de la documentation actuellement disponible.

\section{LA TOMBE DE FONT DE LA VIE}

\section{LE SITE}

Le village de Saint-Bauzille-de-Montmel se trouve entre Montpellier et Alès, à $20 \mathrm{~km}$ au nord-nord-est de la première ville et à $25 \mathrm{~km}$ de la Méditerranée, dans un petit bassin au contact des basses et moyennes Garrigues drainé par de petits affluents de la Bénovie, rivière tributaire du Vidourle (fig. 1). Une imposante colline calcaire, synclinal perché culminant à $273 \mathrm{~m}$ d'altitude, le Puech des Mourgues ou Pic Saint-Léon, le domine, au nord, d'une dénivellation de plus de $160 \mathrm{~m}$ (fig. 2 et 3 ). Elle constitue, avec ses voisines du massif du Bois de Paris au nord-est et de la Suque au sud-ouest, la première ligne de relief important depuis la région littorale, bien visible depuis la côte méditerranéenne.

La partie sommitale du Puech des Mourgues forme un plateau entouré d'une double couronne de falaises verticales de calcaire miroitant du Valanginien supérieur. Celles-ci surplombent un versant d'abord calcaire puis marneux (Valanginien inférieur), dont la pente s'atténue vers le bas. C'est sur le versant méridional de cette colline, en contrebas des falaises et à $750 \mathrm{~m}$ au nord-nord-ouest du centre du village, dans le quartier de Font de la Vie, qu'a été découverte la tombe présentée ici (fig. $2, \mathrm{n}^{\circ} 1$ et fig. 3 ).

\section{LES CIRCONSTANCES DE LA DÉCOUVERTE}

Le versant méridional du Puech des Mourgues fut jadis aménagé en terrasses agricoles. Puis, peu à peu abandonnées, ces terres proches du village sont depuis ces der- nières années progressivement bâties de maisons d'habitation. La sépulture de Font de la Vie a été repérée le 5 août 1993 par Pierre-Yves Genty, ingénieur au Service régional de l'archéologie du Languedoc-Roussillon, au cours d'une journée d'expertise de ce secteur, dans une parcelle autrefois cultivée (oliviers) et qui venait d'être en partie décapée par la lame d'un bulldozer pour aménager un parking ${ }^{1}$. Une petite aire de terre noire enrobant des charbons de bois, des os et des tessons brûlés ainsi que de menus fragments de bronze déformés par le feu, apparaissait à la base du raclage. P.-Y. Genty préleva quelques objets manifestement déplacés par l'engin mécanique sur le dessus de cette aire et à ses abords immédiats et fit les premières observations dans une notice de découverte déposée au Service régional de l'archéologie. Nous pûmes effectuer une fouille de sauvetage dans les jours qui suivirent ${ }^{2}$.

\section{L'ENVIRONNEMENT ARCHÉOLOGIQUE}

Le plateau sommital du Puech des Mourgues, avec ses ruines du monastère de femmes dédié à saint Léon, dont la fondation remonterait à 1223 , ne paraît guère occupé avant le Moyen Âge (Louis, Bruguière, 1932, p. 16). En revanche, un petit habitat protohistorique puis galloromain est attesté sur la partie haute du versant sud-est du Puech, sur la terrasse située au-dessus de la falaise inférieure et en contrebas de la falaise supérieure (Louis, Bruguière, 1932, p. 7-8; Gallia, Informations archéologiques, 33, 1975, p. 509-510) (fig. 2, ${ }^{\circ} 2$ ). Des prospections de surface récentes (B. Dedet puis P.-Y. Genty) indiquent que cet habitat de hauteur pourrait être contemporain de la sépulture de Font de la Vie, dont il n'est distant que de $200 \mathrm{~m}$ : en effet, y furent recueillis, entre autres, des tessons datables des $\mathrm{V}^{\mathrm{e}}$ et $\mathrm{IV}^{\mathrm{e}} \mathrm{s}$. avant J.-C. (amphores étrusque de type 4 - Py, 1985 - et massaliète, céramiques non tournées, grises monochromes et pseudo-ioniennes).

Sur la partie inférieure du versant sud du Puech, M. Louis et $R$. Bruguière ont jadis signalé une station de surface néolithique et deux sépultures à inhumation,

1. Parcelle cadastrale $\mathrm{n}^{\circ} 1521$, section $\mathrm{C}, 2^{\mathrm{e}}$ feuille, appartenant à la commune. Coordonnées Lambert III : $X=730,105 ; Y=3165,060 ; Z=152 \mathrm{~m}$. 2. Nous remercions très vivement Mme Dominique Marchand et Mme Martine Schwaller, conservatrice au Service régional de l'archéologie du Languedoc-Roussillon, qui ont participé à la fouille, M. Pierre-Yves Genty et $M$. Thierry Janin pour leurs informations sur la découverte, et M. Georges Marchand pour les renseignements cadastraux nécessaires. 


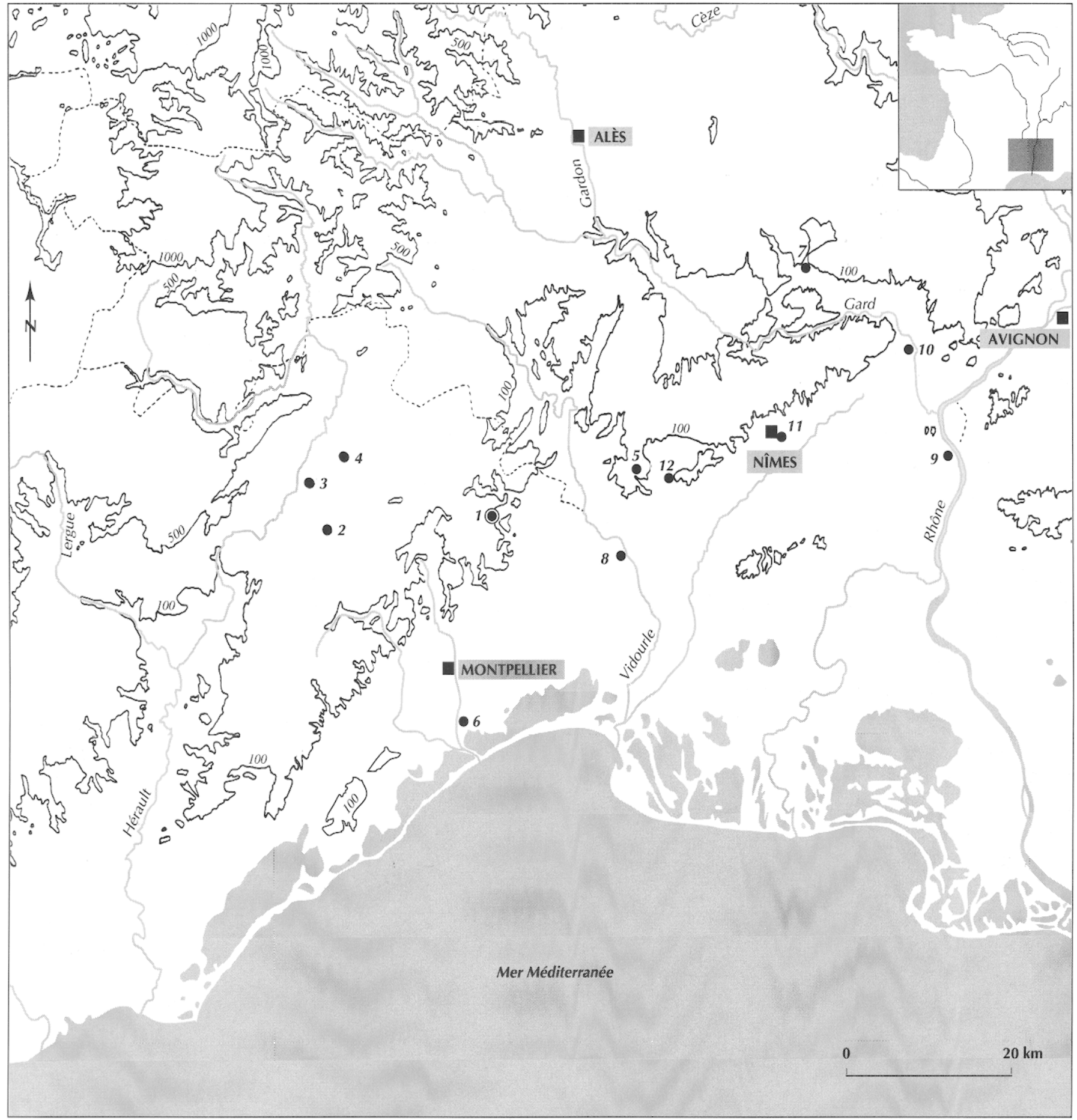

Fig. 1. Situation de la sépullure de Font de la Vie à Saint-Bauzille-de-Montmel (Hérault) parmi les tombes ou nécropoles $d u V T^{e}$ au II $I^{e}$. avant J.-C. du Languedoc oriental.

1. Font de la Vie;

2. Cambous 15 (Viols-le-Fort, Hérault);

3. Frouzet BI (Saint-Martin-de-Londres, Hérault);

4. Ravin des Arcs 6 (Notre-Dame-de-Londres, Hérault);

5. Bergerie Hermet (Calvisson, Gard);

6. la Congourlude (Lattes, Hérault);
7. Préville ou Château-Bérard (Uzès, Gard);

8. Ambrussum-le Sablas (Villetelle, Hérault);

9. les Colombes et le Mas de Jallon (Beaucaire, Gard);

10. Le Marduel, tombe d'Atila (Sernhac, Gard);

11. octroi de Beaucaire (Nîmes, Gard);

12. gare de Nages (Nages-et-Solorgues, Gard). 


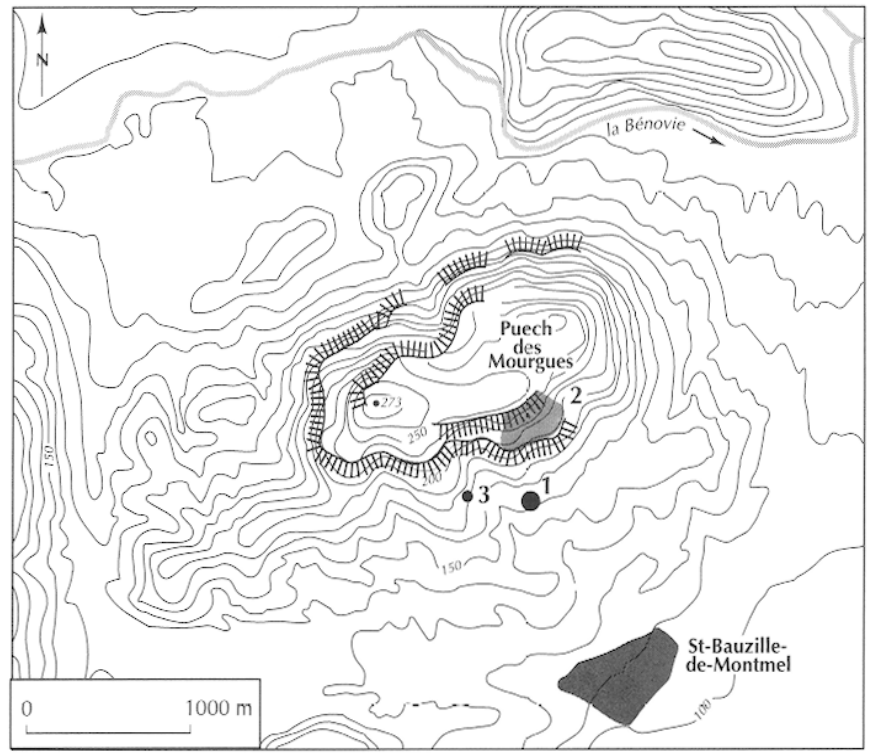

Fig. 2. Le site.

1. sépulture de la Font de la Vie;

2. habitat protohistorique du Puech des Mourgues;

3. emplacement approximatif des sépultures à inhumation découvertes avant 1932. Equidistance des courbes : $10 \mathrm{~m}$.

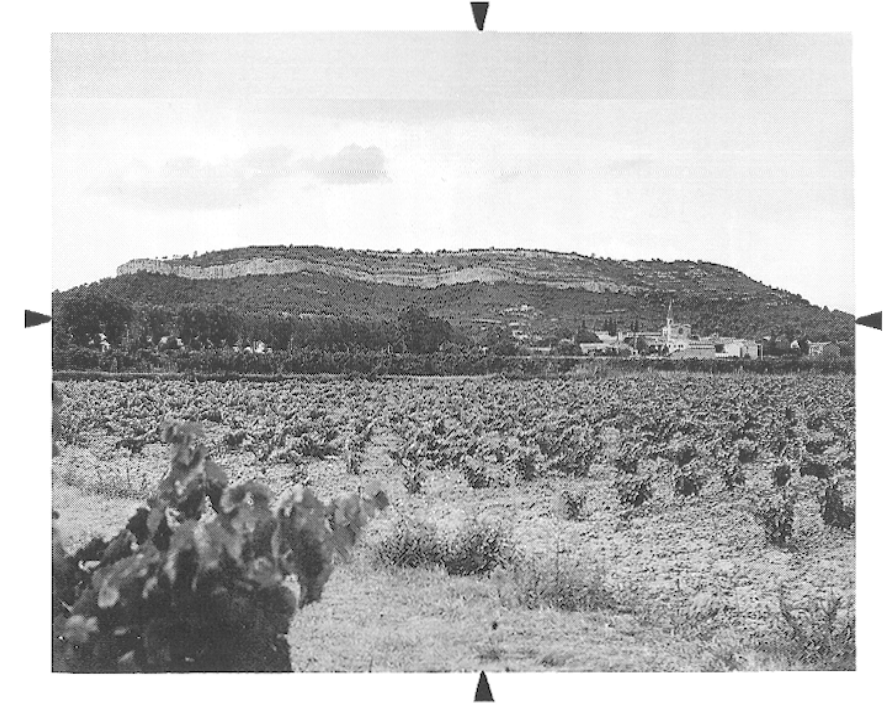

Fig. 3. Vue générale du versant sud du Puech des Mourgues prise du sud.

La sépullure de Font de la Vie se trouve au croisemenl des flèches.

du début de cette période, ont été fouillés dans les environs : Piocam (ou Puech Camp) (Saint-Bauzille-deMontmel) à 1,7 km au nord-est (Louis, Bruguière, 1932, p. 27-28), les Tourelles 1 (Buzignargues) à 2,2 km à l'estnord-est (Fédières, Gasco, 1979), et Pont de la Bénovie (Buzignargues) à 4 km à l'est (Arnal, Jeanjean, 1936-1946, p. 108-110).

\section{DESCRIPTION DE LA TOMBE}

La parcelle où se trouvait la tombe s'étend entre deux thalwegs peu profonds à l'ouest et à l'est, et est limitée au sud, vers l'aval, par un muret de soutènement. La surface décapée par les engins mécaniques couvre environ $1000 \mathrm{~m}^{2}$. Elle forme un rectangle allongé d'environ $50 \mathrm{~m}$ d'est en ouest sur $20 \mathrm{~m}$ du nord au sud, en légère pente, environ $10 \%$, du nord vers le sud. D'après ce que l'on peut observer en bordure de cette aire, l'épaisseur de terre enlevée n'excède pas $10 \mathrm{~cm}$. Le décapage a été effectué jusqu'au substrat rochcux calcaire, dont les bancs les plus proéminents ont été mis à nu ici et là. Ailleurs, subsiste une mince pellicule de terre marron jaune enrobant des éclats de calcaire.

L'aire de terre sombre correspondant à la tombe se trouve près de l'angle nord-ouest du rectangle décapé, à environ $5 \mathrm{~m}$ de ses limites. Sa couleur tranche nettement 
Fig. 4. Coupe de la tombe selon l'axe sud-nord.

1. fosse sépulcrale (u.s. 1);

2. couche de terre non touchée par le bulldozer (u.s. 2);

3. substratum rocheux (u.s. 3);

4. remplissage de la fosse (u.s. 4);

5. dépôt annexe d'un vase tourmé à gros dégraissant (u.s. 5).

En pointillé, surface du terrain avant le décapage par l'engin mécanique.

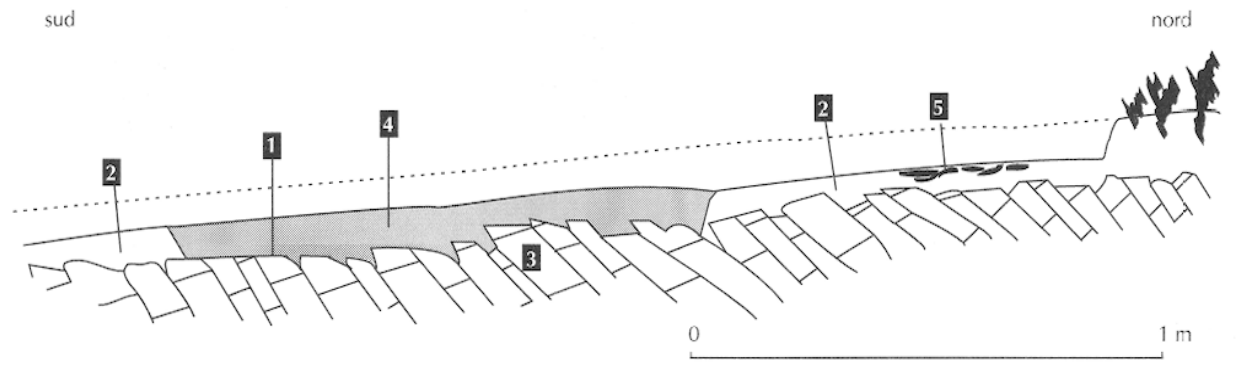

sur celle de la terre brune beaucoup plus claire qui l'environne. Son plan est grossièrement arrondi et son diamètre moyen de $1,10 \mathrm{~m}$. La fouille permet de constater qu'il s'agit du comblement d'une petite fosse (u.s. 1), creusée dans la couche de terre brun clair (u.s. 2) jusqu'au substratum rocheux (u.s. 3). Ce dernier, en pente générale vers le sud, formé de strates calcaires relevées, est très irrégulier. Les bords de la fosse sont abrupts (fig. 4). Le remplissage de celle-ci (u.s. 4) est composé d'un sédiment gris noir incluant de rares charbons de bois, des éclats de calcaire, des os humains incinérés, des tessons portant des traces d'un violent feu secondaire et de menus morceaux d'objets de bronze souvent déformés par la chaleur ou présentant un début de fusion. Ces éléments ont le plus souvent des dimensions infimes, le tamisage de l'ensemble du remplissage de la fosse ayant été effectué à l'eau, à la maille $1 \mathrm{~mm}$. Ce sédiment s'insinue dans les moindres recoins et interstices du rocher qu'il a teinté d'une couleur noirâtre charbonneuse. Son épaisseur, qui n'excède pas $10 \mathrm{~cm}$, est toutefois très variable en raison des irrégularités du substratum. Mais sans doute n'a-t-on ici que la partie inférieure de la fosse (sans qu'il soit possible d'évaluer la part de ce qui a disparu). De toute évidence, ce matériau de comblement u.s. 4 provient d'un bûcher funéraire, ce dernier étant situé dans un autre lieu.

À l'extérieur de la fosse, à $0,50 \mathrm{~m}$ de son bord septentrional, dans la couche de terre brun clair (u.s. 2) non touchée par les engins mécaniques, a été découverte une portion d'urne tournée à gros dégraissant écrasée sur place, sur une superficie d'un quart de mètre carré environ (u.s. 5) (fig. 4). Ces tessons ne portent aucune trace de feu secondaire. La proximité de ces vestiges avec la fosse sépulcrale, dans une couche par ailleurs vierge de tout document archéologique, indique qu'ils font partie de la tombe et en constituent un dépôt annexe.

Quelques éléments portant, sauf une exception, les stigmates du passage sur le bûcher ont été retrouvés sur le dessus de la surface décapée, aux abords immédiats de la fosse sépulcrale u.s. 1, ainsi qu'en trois points un peu plus éloignés :

- à $4 \mathrm{~m}$ à l'ouest du bord occidental de la fosse, un fragment crânien incinéré et une pointe de lance en fer à laquelle adhèrent encore, collés par la corrosion, des éléments ligneux ou osseux brûlés;

- à $2 \mathrm{~m}$ à l'est du bord oriental de la fosse, un morceau non brûlé d'anse de coupe attique;

- à 5,50 m plus à l'est, un autre morceau non brûlé de la même anse, ainsi que trois fragments brûlés de coupe attique, quelques menus morceaux d'objets en bronze déformés par le feu et trois esquilles d'os humains incinérés.

Ces trois points sont situés dans le même alignement qui est celui du sens de travail de l'engin mécanique. Nul doute que tous ces éléments proviennent de la fosse u.s. 1 et qu'ils ont été déplacés par le bulldozer. Par ailleurs, aucun vestige pouvant provenir de la sépulture n'a été décelé lors de l'examen des déblais accumulés par l'engin mécanique sur les bords du secteur dégagé.

Aucun autre objet ou trace de sédiment de couleur différente de celle de la terre brun clair surmontant le rocher n'est apparu ailleurs sur le reste de la surface décapée. De nombreux petits sondages ont été effectués jusqu'au substratum rocheux, toujours très proche, dans les secteurs où celui-ci n'était pas visible. Aucun n'a révélé de document archéologique. Les environs immédiats de l'aire déblayée, au nord et à l'ouest, n'ont pas non plus livré de vestige en surface, mais le couvert végétal masque souvent le sol. Cette tombe paraît donc isolée, mais il est impossiblc d'ĉtrc formel sur ce point. 


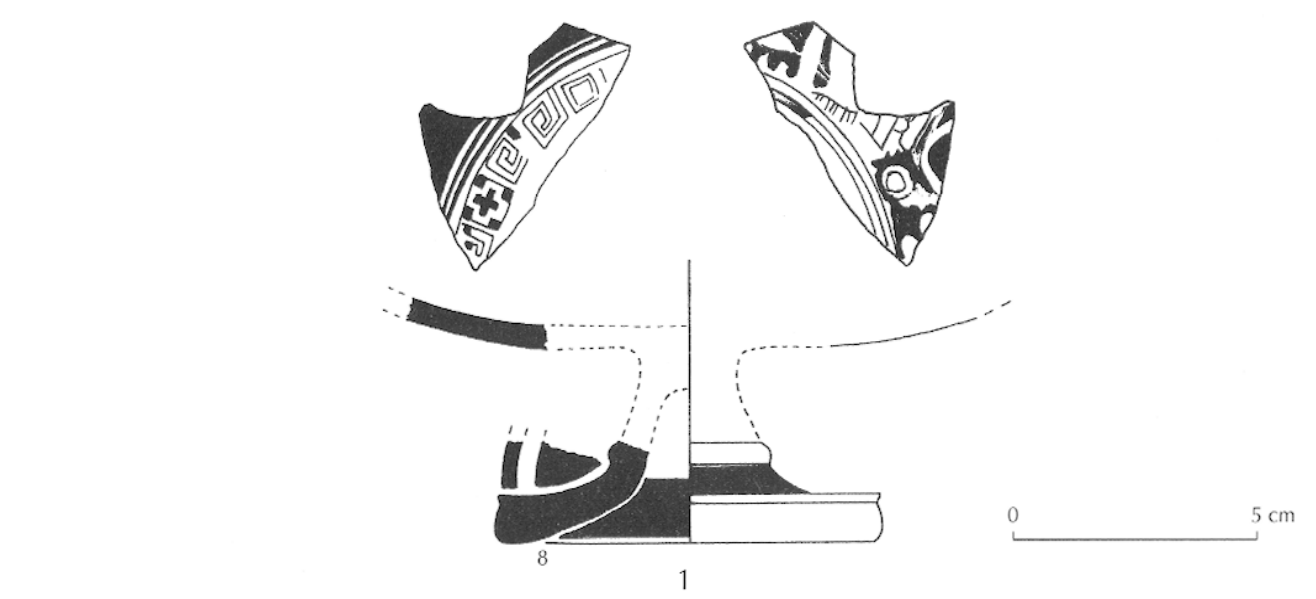

Fig. 5. Mobilier de la fosse sépulcrale u.s. I;

1. coupe attique à figures rouges de type $C$;

2. coupe attique à figures rouges à pied sans tige de type

" inset lip " (2a: face extérieure avec décor de palmette;

$2 b$ : anse avec panneau réservé).

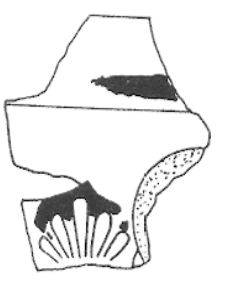

$2 \mathrm{a}$

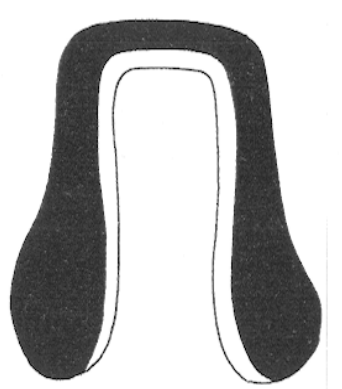

$2 \mathrm{~b}$

\section{LE MOBILIER DE LA TOMBE}

\section{Le mobilier de la fosse sépulcrale U.S. 1}

\section{La céramique}

Le mobilier céramique de la fosse sépulcrale u.s. 1 se compose d'une trentaine de tessons de céramique attique à figures rouges portant, sauf exception, les traces du passage sur le bûcher. D'après le profil des secteurs conservés et la différence de couleur de pâte des tessons, il semble que nous ayons affaire à deux coupes, l'une représentée par sa partie inférieure, l'autre par sa partie supérieure ${ }^{3}$.

3. Cette hypothèse de la présence de deux coupes, plutôt que celle d'un seul récipient, résulte d'une discussion avec $\mathrm{M}^{\text {me }}$ Daniela Ugolini à qui
1. Coupe attique à figures rouges de type $\mathrm{C}$ de Bloesch dont il ne subsiste que le pied ( 9 tessons) et le fond de la vasque (quatre tessons) (fig. 5). Le pied (diamètre $: 8 \mathrm{~cm}$ ) a la forme d'un disque horizontal épais, mouluré sur sa tranche; le replat horizontal est séparé de la partie supérieure du pied par un bourrelet peu marqué. Les traces de feu secondaire que présentent les tessons de ce vase ne sont pas homogènes : la couleur de la pâte varie du beige rosé au gris rosé et au gris. Sur deux tessons jointifs de la partie inférieure de la vasque, on distingue quelques éléments du décor : sur la face intérieure, bordure de médaillon avec grecque à méandres vers la droite interrompue par un rectangle cantonné d'une croix; sur la

nous adressons tous nos remerciements pour ses remarques sur la céramique attique. 
face extérieure peut-être base de draperie avec détails incisés ou peints avec un instrument très fin, et enroulement de volute (?), sur un filet réservé. Les coupes $\mathrm{C}$ sont datées entre 520 et 430 (Bloesch, 1940, p. 145). La forme du pied du spécimen de Font de la Vie est proche de l'exemplaire Agora ${ }^{\circ} 413$ considéré comme l'un des plus tardifs à l'agora d'Athènes, entre 480 et 450 (Sparkes, Talcott, 1970, I, p. 91 et 264; II, fig. 4 ; Bloesch, 1940, pl. $35,3 \mathrm{~b})$. Les motifs de la bordure du médaillon sont bien connus dans les trois premiers quarts du $\mathrm{V}^{\mathrm{e}} \mathrm{s}$. chez des peintres des époques " archaïque tardive ", "classique ancienne » et « classique» de J. Boardman ${ }^{4}$.

2. Coupe attique à figures rouges à pied sans tige de type " inset lip» (Sparkes, Talcott, 1970, I, p. 101-102, $\mathrm{n}^{\circ}$ 469-473) (fig. 5). Très incomplète également, elle n'est représentée que par dix-sept tessons appartenant au bord, à la partie supérieure de la vasque et aux deux anses. Le bord (diamètre à l'ouverture de l'ordre de $20 \mathrm{~cm}$ ) est fin et évasé. Il présente à l'extérieur, une concavité peu marquée, formant une carène avec la panse; il possède à l'intérieur un ressaut, situé un peu plus bas que la carène extérieure. Les anses sont horizontales et relevées. À l'exception notable d'une anse échappée au bûcher et non brûlée, tous les tessons portent les traces d'un feu secondaire violent : la pâte est de couleur gris sombre très soutenu et homogène. Le vernis noir ne subsiste plus que par endroits. Une palmette réservée est visible à l'extérieur près d'un arrachement d'anse (fig. 5, $\mathrm{n}^{\circ} 2 \mathrm{a}$ ). Les anses ont des panneaux réservés (fig. $5, \mathrm{n}^{\circ} 2 \mathrm{~b}$ ). Cette forme de coupe à pied sans tige de type "inset lip " est créée dans le deuxième quart du $\mathrm{V}^{\mathrm{e}} \mathrm{s}$. et persiste jusqu'au premier quart du IV e s. (Sparkes, Talcott, 1970, I, p. 102). La forme de l'exemplaire de Font de la Vie est semblable à Agora $n^{\circ} 471$, daté entre 470 et 450 (Sparkes, Talcott, 1970, I, p. 268, et II, fig. 5).

\section{Les objets en fer}

3. Pointe de lance à douille, flamme en forme de feuille de laurier à nervure centrale de section losangique (fig. 6). Extrémité de la douille incomplète; extrémité de la pointe

\footnotetext{
4. Boardman, 1975, par exemple $n^{\circ} 245,248$ ou 253 (coupes du peintre de Brygos) ; Boardman, 1989, par exemple n ${ }^{\circ} 73-2$ (coupe du peintre de Tarquinia), $n^{\circ} 80-2$ et 84 (coupes du peintre de Penthesilée), ou $n^{\circ} 90$ (coupe du peintre de Curtius). D'autres exemples à Aléria dans les tombes 85, 90, 91 et 92 (Jehasse, 1973, n ${ }^{\circ} 1563$, p. 406 et pl. $59 ; \mathrm{n}^{\circ} 1771$, p. $446-447$ et pl. $60 ; n^{\circ} 1837$, p. 459 et pl. $63 ; n^{\circ} 1904$, p. 473 et pl. $57-$ $58 ; n^{\circ} 2025$, p. 493 et pl. 56 ).
}

tordue. Longueur conservée : $113 \mathrm{~mm}$; largeur maximum conservée : $28 \mathrm{~mm}$. Des fragments de charbon de bois et d'os incinéré sont accrochés à la pièce par oxydation.

4. Fragment de tige recourbée de section sublosangique : pourrait appartenir à un arc de fibule (type non déterminable) (fig. 6). Longueur conservée : $27,5 \mathrm{~mm}$; épaisseur maximum : 5 sur $4,5 \mathrm{~mm}$.

\section{Les objets en bronze}

5. Fragment de garniture et système d'attache de courroie, comprenant trois éléments (fig. 6) :

- extrémité d'un bandeau de tôle de $11 \mathrm{~mm}$ de large et $0,5 \mathrm{~mm}$ d'épaisseur, conservé sur $12 \mathrm{~mm}$ de longueur, décoré sur les bords d'un rang de perles au repoussé; l'extrémité est perforée sur son axe pour permettre la fixation d'une boucle;

- la boucle, longue de 21,8 mm, est fortement élargie et aplatie au niveau des attaches, perforées également;

- un rivet, de $10 \mathrm{~mm}$ de long et de $2 \mathrm{~mm}$ de section, maintient le tout; sa longueur est compatible avec la fixation d'une courroie de cuir.

6. Fragment de ruban déformé par le feu (fig. 6) : pourrait appartenir à un bracelet-armille; section ovalaire $(4,6$ sur 2,8 $\mathrm{mm})$; longueur conservée : $14 \mathrm{~mm}$.

7 et 8. Deux anneaux semblables, fragmentés, coulés dans un moule bivalve (fig. 6); section lenticulaire; rainure parallèle à chaque bord sur chacune des deux faces; diamètre : $35 \mathrm{~mm}$; largeur du jonc, respectivement : 7,2 et $7,5 \mathrm{~mm}$; épaisseur de la tige, respectivement, 3,9 et $3,6 \mathrm{~mm}$; traces de feu secondaire; un fragment de bronze déformé par la chaleur est collé contre un morceau d'un des anneaux.

9. Trois fragments d'un anneau elliptique (fig. 6) ; diamètre : de 32,5 à $38 \mathrm{~mm}$; tige lisse de section circulaire (8,4 mm d'épaisseur) ; traces de feu secondaire.

Fig. 6. Mobilier de la fosse sépulcrale u.s. $l$; 3. fer de lance;

4. tige en fer (fragment de fibule?); 5. garniture et attache, en bronze, de courroie; 6. tige en bronze (fragment de bracelet-armille?); $7 \grave{a} 12$ et 14. anneaux en bronze; 13. maillon de chaînette en bronze; 15. coupelle ou simpulum en tôle de bronze;

16. manche d'ustensile en bronze; 17-18. plaque en tôle de bronze (éléments de parure ou d'habillement); 19 à 21. jetons en bronze; 22-23. anneaux en os. 

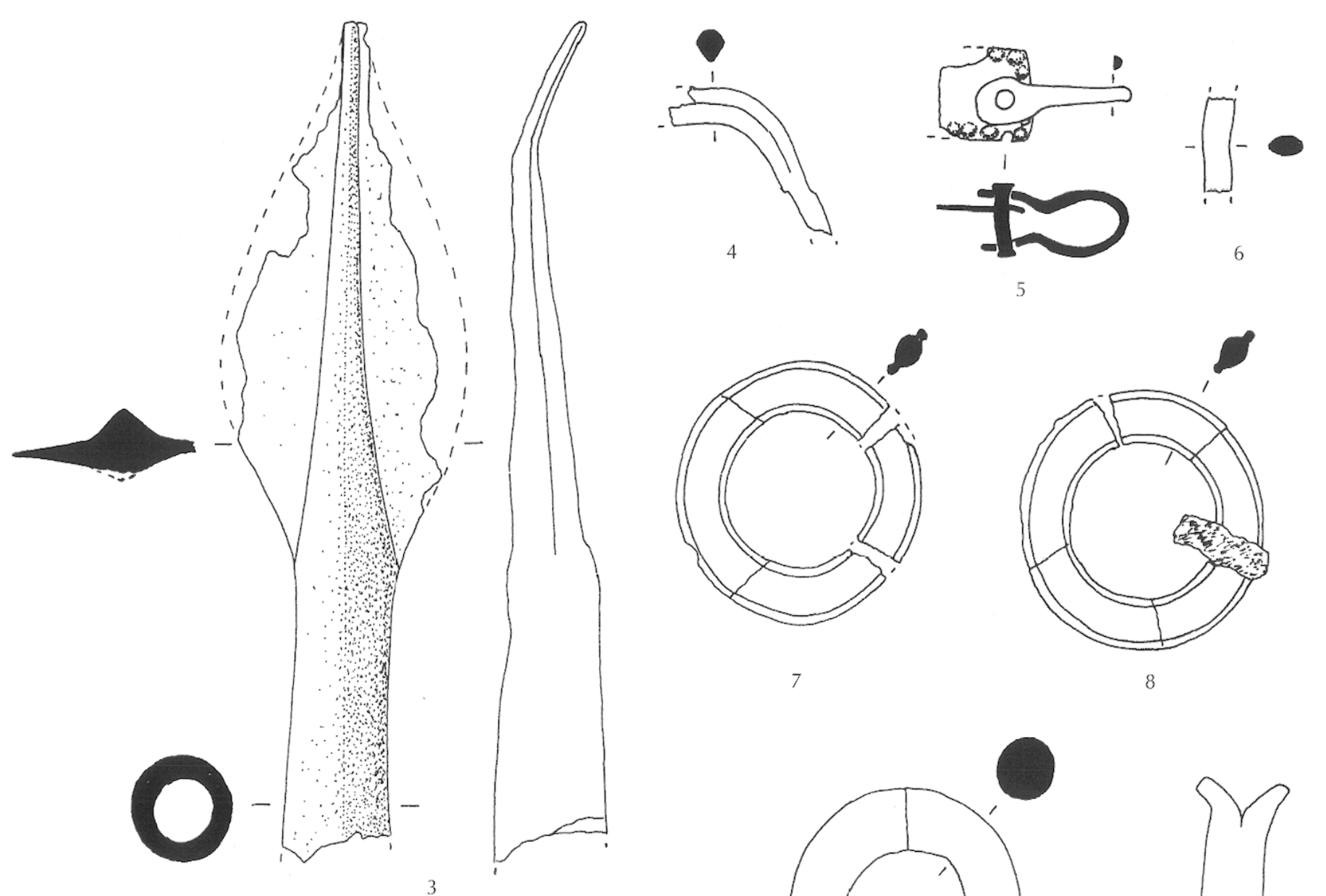

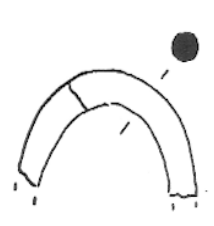

10

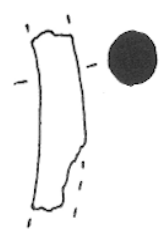

11

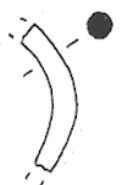

12

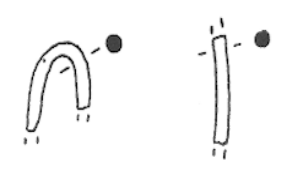

13

14
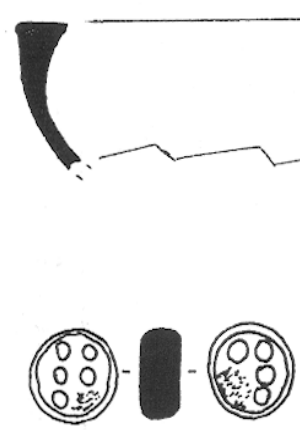

19

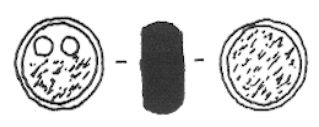

20

(2) -1

21

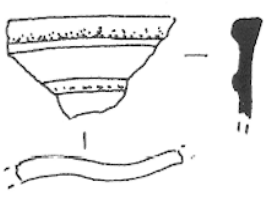

17

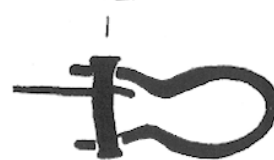

5

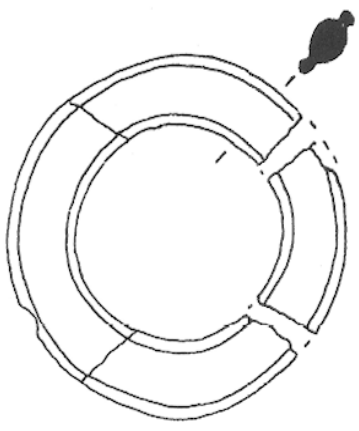

7

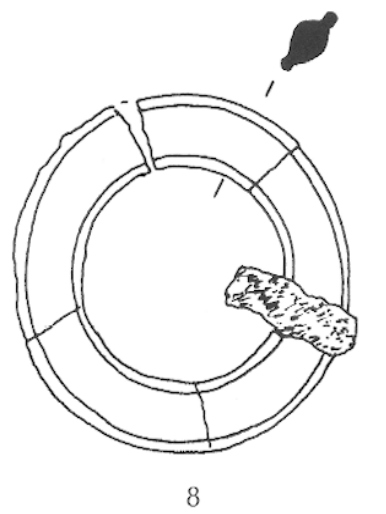

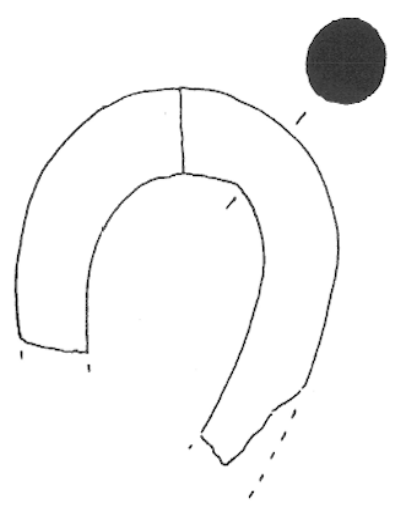

9

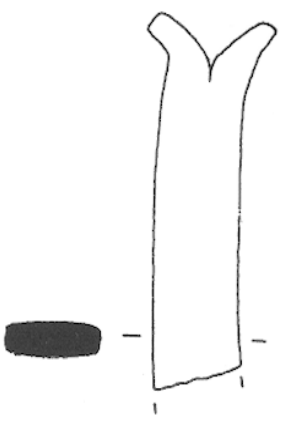

16

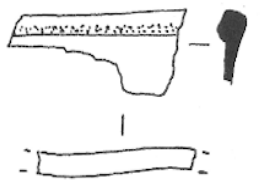

18

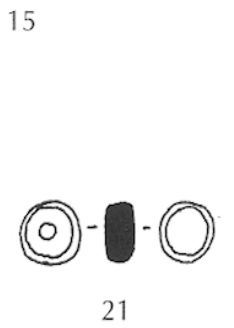

$3 \mathrm{~cm}$

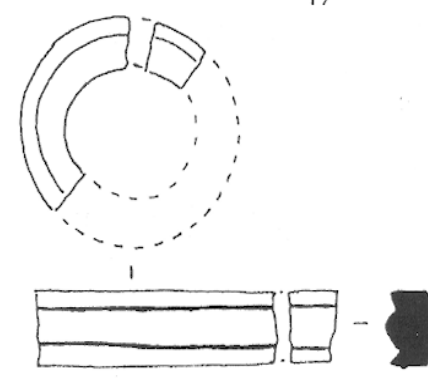

22

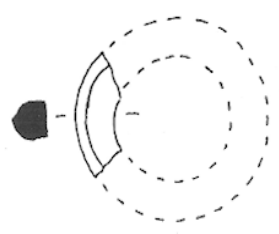

23 
10. Deux fragments d'un petit anneau déformé par le feu (fig. 6) ; diamètre de l'ordre de $20 \mathrm{~mm}$; tige de section circulaire de $3 \mathrm{~mm}$ de diamètre.

11. Fragment d'anneau (fig. 6), de section circulaire de $5 \mathrm{~mm}$ d'épaisseur; longueur conservée : $17 \mathrm{~mm}$.

12. Fragment d'anneau (fig. 6), de section circulaire de $2,5 \mathrm{~mm}$ d'épaisseur.

13. Fragment de fil recourbé, peut-être maillon de chaînette (fig. 6) ; section circulaire de 1,5 mm d'épaisseur.

14. Fragment de fil de section circulaire (fig. 6) (épaisseur : $1,5 \mathrm{~mm}$ ).

15. Fragment de coupelle arrondie convexe, ou de simpulum (fig. 6); diamètre à l'ouverture : $9 \mathrm{~cm}$; bord épaissi en marli horizontal; épaisseur de la paroi, $5 \mathrm{~mm}$ au bord, 1,3 $\mathrm{mm}$ sur la panse.

16. Fragment de manche d'ustensile (cuiller? simpulum ? passoire?) (fig. 6), à extrémité proximale découpée en deux languettes incurvées; section rectangulaire de $3,7 \mathrm{~mm}$ d'épaisseur; longueur conservée : $36,5 \mathrm{~mm}$; largeur : $9 \mathrm{~mm}$.

17. Fragment de tôle déformé par le feu, présentant un bord épaissi et une nervure parallèle au bord (fig. 6) ; longueur conservée : $17,5 \mathrm{~mm}$; épaisseur : de 1 à $2,5 \mathrm{~mm}$.

18. Fragment de tôle déformé par le feu, à bord épaissi (fig. 6) ; longueur conservé : $16,5 \mathrm{~mm}$; épaisseur : 1,2 à $2,5 \mathrm{~mm}$.

19. "Jeton» circulaire, portant sur chacune des faces six cercles incisés, rangés sur deux files (fig. 6) ; diamètre : $8,5 \mathrm{~mm}$; épaisseur : $4,1 \mathrm{~mm}$; poids : $1,52 \mathrm{~g}$. Nous qualifions cet objet de "jeton ", de même que les deux suivants, mais peut-être s'agit-il de poids?

20. «Jeton» semblable au précédent (fig. 6), mais la corrosion ne permet de discerner que deux des cercles incisés; diamètre : $9 \mathrm{~mm}$; épaisseur : $4,5 \mathrm{~mm}$; poids : $1,40 \mathrm{~g}$.

21. "Jeton" plus petit que les deux autres, avec un seul cercle central incisé, visible sur la face la moins corrodée (fig. 6) ; diamètre : $6,1 \mathrm{~mm}$; épaisseur : $3,1 \mathrm{~mm}$; poids : $0,46 \mathrm{~g}$.

Nombreux fragments de tôle de bronze de 0,3 à $0,6 \mathrm{~mm}$ d'épaisseur, souvent repliés en double épaisseur (non figurés). Poids total : $36,5 \mathrm{~g}$. Tous ces fragments sont déformés par le feu; deux d'entre eux présentent un début de fusion. D'après l'absence de décor il semblerait que ces éléments se rapportent plutôt à un récipient (coupe? simpulum?).

Nombreux fragments d'objet en bronze, en fusion ou présentant un début de fusion, notamment des éléments

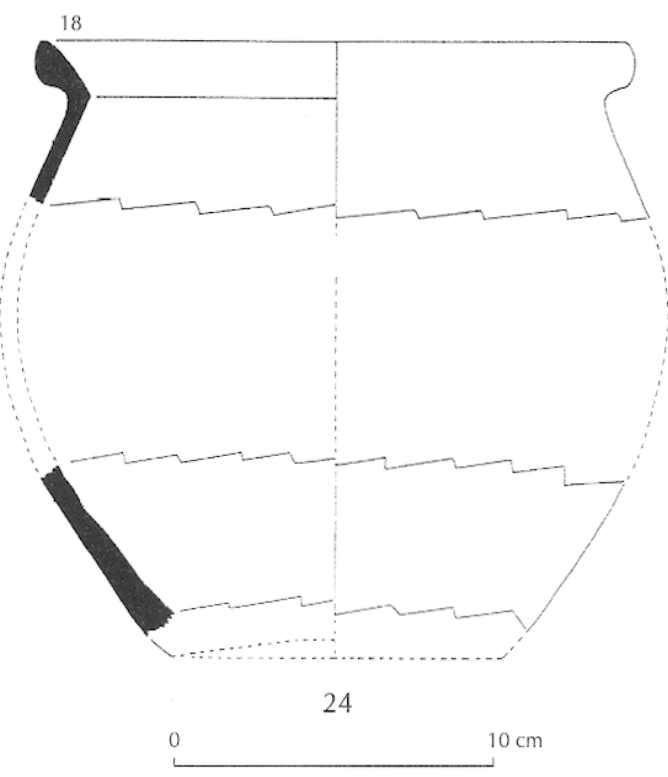

Fig. 7. Ume en céramique $\left(n^{\circ} 24\right)$ tournée à gros dégraissant du dépôt annexe u.s. 5.

de tôle de 0,6 à $1,2 \mathrm{~mm}$ d'épaisseur (non figurés). Poids total : $131 \mathrm{~g}$.

\section{Les objets en os}

22. Deux fragments d'un anneau, brûlé (fig. 6); la face extérieure est moulurée : deux rainures profondes dégagent un tore central proéminent; les faces supérieure et inférieure sont planes; diamètre : $22 \mathrm{~mm}$; hauteur : 7,2 $\mathrm{mm}$; épaisseur : $5,5 \mathrm{~mm}$.

23. Fragment d'un petit anneau, brûlé (fig. 6); face extérieure arrondie; faces supérieure et inférieure planes ; diamètre : $1,8 \mathrm{~mm}$; hauteur : $3,8 \mathrm{~mm}$; épaisseur : $4,1 \mathrm{~mm}$.

\section{Le mobilier déposé aux abords de la fosse sépulcrale (U.S. 5)}

24. Urne fragmentaire en céramique tournée à gros dégraissant de forme 1 (fig. 7) (Dedet, 1978) ${ }^{5}$ : col divergent très bas, séparé de la panse à l'intérieur par une arête vive; lèvre à profil arrondi vers l'extérieur; diamètre à l'ouverture : $18 \mathrm{~cm}$; pâte de couleur ocre à gris en surface et en épaisseur. Le dégraissant, très abondant et très

5. Correspond à la production dénommée " céramique commune tournée du Languedoc oriental " dans Py dir., 1993, p. 161-162. 
mal calibré (jusqu'à 4,5 mm), est constitué surtout de particules de quartz roulées. Ce type de céramique est attesté en Languedoc oriental dans le seconde moitié du Ve $s$. avant J.-C. et la première moitié du siècle suivant, avec cependant un dégraissant à base de calcite broyée et non de quartz (Dedet, 1978). Une production similaire, mais à inclusions à base de quartzite cette fois, dénommée " céramique de cuisine tournée ", a été mise en évidence à Béziers dans les trois derniers quarts du $\mathrm{V}^{\mathrm{e}} \mathrm{s}$. avant J.-C. (Ugolini, Olive et alii, 1991, p. 167-171).

\section{Bilan du mobilier funéraire}

L'état de conservation de tout ce mobilier, qui résulte du rituel mis en œuvre, avec passage des objets sur le bûcher, et sans doute aussi des travaux agricoles et des conditions de découverte, ne permet pas de dresser la liste complète du matériel qui a été déposé dans la fosse sépulcrale ou ses abords. Nombreux sont les points d'interrogation. Tentons cependant un bilan de ce qui a accompagné le défunt lors de la crémation et dans la fosse sépulcrale (fig. 5 et 6 ) :

- parure et habillement : une courroie garnie de bronze $\left(n^{\circ} 5\right)$, des anneaux en os ayant sans doute fait partie d'un collier ou d'un pendentif ( $\mathrm{n}^{\circ} 22$ et 23 ), plusieurs anneaux et un élément de chaîne, en bronze, participant soit de l'habillement soit de la parure ( $\mathrm{n}^{\circ} 7$ à 14$)$, peut-être une fibule en fer (?) $\left(n^{\circ} 4\right)$, peut-être un bracelet-armille en bronze (?) $\left(n^{\circ} 6\right)$, peut-être un ou plusieurs objets de parure ou d'habillement comprenant des éléments en tôle de bronze (?) ( $\mathrm{n}^{\circ} 17-18$ et fragments non figurés);

- arme : une lance à pointe en fer $\left(n^{\circ} 3\right)$;

- ustensiles divers : deux coupes attiques $\left(n^{\circ} 1\right.$ et 2$)$, une coupelle ou un simpulum en bronze $\left(n^{\circ} 15\right)$, un objet non déterminé pourvu d'un manche (peut-être celui du simpulum précédent?) $\left(\mathrm{n}^{\circ} 16\right)$, sans doute un autre récipient en tôle de bronze (éléments non figurés) et trois jetons ou poids en bronze ( $n^{\circ} 19$ à 21$)$.

À cela s'ajoute le dépôt, extérieur à la fosse sépulcrale, d'une urne ou portion d'urne (contenant éventuellement une offrande périssable) (fig. 7). Mais là aussi, comme pour la fosse sépulcrale, des éléments ont pu disparaître par suite des travaux agricoles et de l'arasement de la partie supérieure de la tombe.

\section{DATATION DE LA SÉPULTURE DE FONT DE LA VIE}

Plusieurs éléments du mobilier permettent de dater l'enfouissement de la tombc au milicu du $\mathrm{V}^{\mathrm{e}} \mathrm{s}$. avant J.-C.
Les deux coupes attiques à figures rouges tout d'abord. Datée en Grèce entre 520 et 430 , la coupe de type $\mathrm{C}$ est très largement exportée sur les sites côtiers du Sud de la France et de Catalogne au premier quart du $\mathrm{V}^{\mathrm{c}}$ s., notamment à Arles, la Monédière, Montlaurès, Ruscino ou Ampurias (Rouillard, 1992, p. 182-184). L'exemplaire de Font de la Vie, tant par sa forme que par ses motifs et son style décoratif, doit être rapporté au second quart de ce siècle. C'est au même quart de siècle que l'on peut placer, sur des critères de forme, la coupe de type "inset lip ", à pied sans tige, de la même tombe (pour les deux coupes voir supra). Ce type de récipient est très abondant dès le deuxième quart du $\mathrm{V}^{\mathrm{e}} \mathrm{s}$. sur de nombreux sites des bords du golfe du Lion dont Marseille (Gantès, 1992, p. 174), Béziers (renseignement de Mme Daniela Ugolini), Arles, Ampurias, Penya del Moro (Sant Just Desvern, Barcelone) (Rouillard, 1992, p. 184), Puig de Benicarlo (Castellon) (Sanmartí Grego, 1976, p. 220222). Il y reste présent, quoique avec des fréquences moindres, jusqu'à la fin du siècle.

La céramique tournée à gros dégraissant, en Languedoc oriental, apparaît surtout dans les habitats de l'arrière-pays mais toujours dans des proportions très faibles, dès le milieu du $V^{e} s$. avant J.-C. et dure jusqu'au début du siècle suivant. Ses meilleurs scores de représentation se situent toutefois au milieu et dans le troisième quart du Ve s. : ainsi, à Vié-Cioutat (Mons, Monteils, Gard), cette catégorie passe de $8 \%$ de l'ensemble du matériel céramique au début de la seconde moitié du $\mathrm{V}^{\mathrm{e}} \mathrm{s}$., à moins de $1 \%$ à la fin du siècle; même diminution dans le même laps de temps au mont Cavalier à Nîmes, de $4 \%$ du total des tessons (sondage 2 , couche 3 ) à $0,5 \%$ (sondage 2, couche 2) (Dedet, 1978, p. 27-31). À Béziers, on note une apparition plus précoce semble-til de la céramique de "cuisine tournée ", dans le deuxième quart du $V^{e}$ s. Elle y est plus abondante que son homologue en Languedoc oriental (13\% du total des vases), et elle y remplace pratiquement la vaisselle culinaire non tournée (Ugolini, Olive et alii, 1991, p. 167 et 192).

Les « jetons » en bronze à cercles incisés sont exceptionnels dans la région. Nous n'en connaissons qu'un exemplaire, semblable aux deux plus grands de Font de la Vie (fig. 7, n ${ }^{\circ} 19$ et 20), dans une couche de la seconde moitié du V $\mathrm{V}^{\mathrm{e}}$ s. de l'oppidum de Vié-Cioutat qui a aussi livré de la céramique tournée à gros dégraissant (Dedet, 1973, p. 37 , fig. 31 , et p. 39 , fig. $33, n^{\circ} 323$, et p. 41 ).

Les autres pièces du mobilier n'apportent pas d'élémont de datation précis. Une mention particulière doit 
toutefois être faite pour le fragment de récipient en bronze à bord épaissi, coupelle ou simpulum (fig. $6, \mathrm{n}^{\circ} 15$ ). Un simpulum à bord épaissi de forme très proche figure dans la tombe 2 de Saint-Julien à Pézenas, Hérault, datée du troisième quart du VIe s. avant J.-C. (Giry, 1965, p. 127). Les coupelles en bronze à bord épaissi sont présentes en Languedoc oriental ou sur les Grands Causses dans des contextes plus anciens, du premier Âge du Fer, ainsi dans les tumuli Cazevieille A5 (Hérault) (Dedet, 1992, p. 374, fig. 119) et Roumagnac 1 à Sévérac-le-Château (Aveyron) (Gruat, Marty, 1993, p. 87-88), ou supposés tels comme dans le tumulus du col de la Vache sur la Can d'Artigues (Lozère) (Hugues, 1960, p. 70 et 72; Dedet, à paraître). Si la durée de l'usage d'un tel objet ne peut être appréciée correctement, vu la rareté des sépultures du milieu de l'Âge du Fer connues en (auule méditerranéenne, sa présence à Font de la Vie, à une époque plus récente, mérite d'être soulignée.

\section{LE DÉFUNT}

En tout, 54,7 g d'ossements humains incinérés ont été recueillis. D'après la morphologie des os du crâne, épaisseur des parois de la voûte, synostose de la suture sagittale et la fermeture de l'apex de la seule dent attestée, il s'agit d'un sujet adulte. En l'absence de doublon, il semble que nous ayons affaire à un seul individu. Les pièces sont très fragmentées. Les morceaux de diaphyses sont très souvent fissurés en ondes concentriques, indice d'une incinération d'os frais (et non d'une incinération tardive d'os secs). Les couleurs varient du noir brillant (rare), au gris bleuté et au blanc (le plus fréquent), mais ces trois teintes peuvent être observées dans la même région du squelette, voire, pour deux d'entre elles, sur le même fragment osseux (par exemple noir en face endocrânienne et blanc en face exocrânienne sur un élément de pariétal). Selon le barème de E. Bonucci et G. Grazziani (Bonucci, Grazziani, 1975) la température de la crémation aurait oscillé, selon les endroits, entre 300$350^{\circ} \mathrm{C}$ et plus de $650^{\circ} \mathrm{C}$.

Toutes les parties du squelette du mort ont été concernées par le prélèvement sur le bûcher funéraire. Peu de pièces cependant peuvent être identifiées avec précision. Celles-ci se répartissent de la manière suivante :

- crâne (poids total : 4,0 g) : un fragment de pariétal avec suture sagittale en cours de synostose et six autres très petits morceaux de voûte crânienne assez épaisse; un fragment d'écaille de temporal; un fragment de maxillaire; un fragment de maxillaire ou de mandibule; une partie inférieure de racine de dent avec apex fermé, à laquelle adhère un fragment de bronze en cours de fusion;

- tronc (poids total : $1,1 \mathrm{~g}$ ) : trois fragments de côtes et un de coxal ;

- membres (poids total : 27,2 g) : un fragment de diaphyse d'humérus; deux fragments de diaphyse de fémur; deux fragments jointifs de diaphyse de fibula; cinquantedeux fragments de diaphyse d'os longs des bras, avantbras, cuisses ou jambes; six fragments de phalanges des mains ou des pieds; neuf fragments de métacarpiens ou de métatarsiens; sept fragments de métacarpiens, métatarsiens ou phalanges;

- fragments indéterminés : deux fragments d'os court ou d'épiphyse $(0,4 \mathrm{~g})$; nombreuses esquilles $(22,0 \mathrm{~g})$.

Ni l'âge au décès, ni le sexe de cet adulte ne peuvent être précisés. La présence d'une arme dans sa tombe, une pointe de lance, constitue toutefois un indice en faveur d'un individu de sexe masculin. D'autre part, la présence de deux coupes attiques, de vaisselle de bronze et d'une parure que l'on soupçonne relativement abondante, pourrait indiquer un personnage important, à l'instar de ce qui se passe dans le groupe des tumulus des Garrigues au VI ${ }^{\mathrm{e}}$ s. avant J.-C. (Dedet, 1995). Mais nous manquons toutefois de points de repères en ce domaine pour le $\mathrm{V}^{\mathrm{e}} \mathrm{s}$. avant J.-C. dans la région.

\section{LES PRATIQUES FUNÉRAIRES MISES EN GUVRE}

Les données de fouille, mais aussi l'état du matériel recueilli, permettent de reconstituer différentes étapes du rituel et des pratiques funéraires en vigueur dans le groupe humain responsable de la sépulture de Font de la Vie au milieu du $\mathrm{V}^{\mathrm{e}} \mathrm{s}$. avant J.-C. On peut distinguer plusieurs actes, globalement selon la succession suivante :

- crémation du cadavre d'un adulte, très probablement un homme d'après son mobilier, sur un bûcher édifié dans un lieu différent de celui où est établie la tombe, et dont aucun vestige n'a été repéré;

- le défunt est accompagné sur le bûcher de son équipement personnel, parures, arme, ustensiles divers dont une des deux coupes attiques (celle de type C), ce qui entraine altérations et déformations des objets, et parfois début de fusion de certaines pièces en bronze;

- l'autre coupe attique (celle de type "inset lip») est, semble-t-il, non pas déposée sur le bûcher mais brisée sur celui-ci. En effet, au contraire de l'ensemble des autres tessons de ce vase, l'une des deux anses ne porte aucun 
stigmate, même léger, de feu secondaire. Peut-être s'agitil de la trace d'une libation effectuée sur le bûcher funèbre avec bris intentionnel du récipient qui a servi à cette action et rejet, hors du foyer, de l'anse par laquelle le vase était tenu ${ }^{6}$

- creusement de la fosse sépulcrale (u.s. 1);

- après la crémation, on recueille, en vrac, une partie des restes du bûcher et l'on place cette masse, magma contenant des restes osseux humains et des morceaux des objets ayant accompagné le corps lors de la crémation, telle quelle, sans contenant, dans la fosse sépulcrale (u.s. 1). Les os ne sont pas mis dans un ossuaire. Le sédiment retrouvé dans la fosse, de même que les dimensions extrêmement réduites de certains débris osseux et mobiliers, montrent bien qu'il n'y a pas eu de prélèvement sélectif ou de tri sur le bûcher refroidi, pas plus que de lavage des restes. On remarquera aussi, à propos de la coupe attique de type "inset lip », que si tous les tessons ne sont pas ramassés, on prend soin de rajouter dans la fosse l'anse qui, lors du bris du vase, avait été préservée de l'action du feu;

- à proximité immédiate de la fosse sépulcrale, on dépose une urne tournée à gros dégraissant (contenant peut-être une offrande périssable), ou un morceau seulement de cette urne (u.s. 5). Ce vase ne porte pas de trace d'un feu secondaire et n'est donc pas passé par le bûcher funèbre. Il possède donc, vis à vis du mort, un statut différent de celui des autres objets de cette tombe, et il intervient peut-être à un autre moment des funérailles : ultime offrande après le dépôt des restes du bûcher dans la fosse sépulcrale?

- bien qu'aucun vestige n'en soit parvenu jusqu'à nous, il va sans dire que la tombe (fosse sépulcrale u.s. 1 et dépôt annexe u.s. 5) a dû posséder un dispositif de couverture. Sans cette protection, l'érosion sur cette pente, même légère, eût tôt fait d'assurer la disparition du dépôt. S'agissait-il d'un tertre de terre, de terre et de. pierres mêlées, ou de pierres seules selon la tradition régionale, d'autant que la pierre abonde dans les environs? Il est impossible de répondre, les pratiques agricoles ayant dû faire disparaître un tel amoncellement bien avant que n'interviennent les engins mécaniques.

\footnotetext{
6. Une telle libation, avec bris dans le bûcher de la coupe utilisée, est attestée en Grèce (Lécrivain, 1896, p. 1378). Jannoray (1955, p. 234 236) estime qu'elle était également pratiquée dans la phase ancienne de la nécropole d'Ensérune actuellement connue (dernier quart du $\mathrm{V}^{\mathrm{e}} \mathrm{s}$. et premier quart du IV $\mathrm{e}$ s. selon les précisions chronologiques apportées récemment par Schwaller, Marchand, 1993, p. 225).
}

\section{LA TOMBE DE FONT DE LA VIE ET L'ÉVOLUTION DES RITES FUNÉRAIRES PROTOHISTORIQUES DU LANGUEDOC ORIENTAL}

À cause de l'indigence de la documentation, il est bien difficile de juger du caractère représentatif ou non de la sépulture de Font de la Vie en Ianguedoc oriental au $\mathrm{V}^{\mathrm{e}} \mathrm{s}$. avant J.-C. En effet, entre le phénomène tumulaire du début du premier Âge du Fer, qui décline très rapidement dès le début du $V^{c} s$. et disparaît à la fin de ce siècle, et les tombes du $\mathrm{I}^{\text {cr }} \mathrm{s}$. avant J.-C., les données sont extrêmement rares, nous l'avons déjà souligné et, de surcroît, dans la plupart des cas, très imprécises. La liste est vite établie : tombes 1, 3 et 4 de la Bergerie Hermet à Calvisson (Gard) et tombe de la Cougourlude à Lattes (Hérault) pour la fin du $\mathrm{V}^{\mathrm{e}}$ ou le début du $\mathrm{V}^{\mathrm{c}} \mathrm{s}$. avant J.-C.; tombe de Préville ou du Château-Bérard à Uzès (Gard) pour la fin du IV ${ }^{\mathrm{c}}$ ou la première moitié du $\mathrm{III}^{\mathrm{e}} \mathrm{s}$. avant J.-C.; sept tombes fouillées à ce jour dans la nécropole du Sablas au pied de l'oppidum d'Ambrussum à Villetelle (Hérault), pour le dernier quart du $\mathrm{III}^{\mathrm{e}}$ et le premier quart du II ${ }^{e}$ s. avant J.-C.; deux à Beaucaire (les Colombes 3 et 4), une à Nîmes, une à Nages et une à Sernhac (toutes dans le Gard), pour le II ${ }^{\mathrm{e}}$ s. avant J.-C. La comparaison des pratiques funéraires que l'on peut déceler dans les quelques tombes de cette période avec celles observées à Font de la Vie apporte cependant dès à présent des informations importantes.

Comparons tout d'abord avec les tumuli du premier Âge du Fer des Garrigues du Gard et de l'Hérault, presque tous antérieurs à la fin du $\mathrm{VII}^{\mathrm{e}}$ s. avant J.-C., ou difficiles à dater précisément à cause de la nature ou de l'indigence du matériel. L'incinération occupe une place quantitativement importante dans cet ensemble, à côté d'autres modes de traitement du corps (dépôt d'une pièce osseuse unique non incinérée, dépôt secondaire après décharnement, possible dépôt primaire). Absente des très rares tumuli plus anciens, ceux de la fin du Bronze final IIIB notamment, elle concerne environ $40 \%$ des défunts aux VIII ${ }^{\mathrm{e}}-\mathrm{VII}^{\mathrm{c}}$ s. Elle est ensuite exclusive, semble-t-il, au VIe s. avant J.-C. : les quatre tumuli des Garrigues connus pour ce siècle sont tous à incinération, Serre des Fontaines 1, vers 600 ou première moitié du $\mathrm{VI}^{\mathrm{e}} \mathrm{s}$., Cambous 15, deuxième quart du VI ${ }^{\mathrm{e}}$ s., Frouzet Bl, deuxième quart ou seconde moitié du VI ${ }^{\mathrm{e}}$ s., Ravin des Arcs 6, Vle s. (Dedet, 1992 et 1995). 
Ia crémation, dans ce contexte culturel des tumuli, a presque toujours lieu dans un endroit différent de celui de la sépulture, mais les modalités sont très différentes de celles que l'on peut observer à la Font de la Vie. Il est très rare que des objets accompagnent le défunt sur le bûcher : jamais les armes, les outils et les ustensiles, ni les poteries. C'est seulement parfois le cas pour des pièces de parure ou d'habillement : $16,6 \%$ des tumulus à incinération seulement sont pourvus de ce type d'objets (Dedet, 1992, p. 153-185). Par ailleurs, lorsque des observations circonstanciées ont été faites, on n'a pas placé dans le tumulus une portion du bûcher funéraire, mais seulement des os brûlés prélevés sur ce bûcher. Il y a donc tri contrairement à la Font de la Vie. Enfin, dans les trois cas, exceptionnels pour la région, de tumulus à incinération sur place (Serre des Cxalères 2, Viols 8 et Frouzet B1, ce dernier seul datable du VI' s.), le matériel accompagnant les restes osseux semble, pour l'essentiel, avoir été déposé après la crémation. Ce n'est qu'à Frouzet B1 qu'une partie de l'équipement du mort, une cnémide et une coupe en bronze, a subi l'action plus ou moins intense d'un feu secondaire, sans cependant atteindre l'état de fusion; les autres pièces du mobilier, notamment celles ressortissant à la parure ou à l'habillement, agrafe de ceinture, boutons ou appliques, bracelet, gisaient dans le tumulus à l'écart de l'aire portant les traces de la crémation.

Les tombes 1, 3 et 4 de la Bergerie Hermet, toutes trois à incinération, appartiennent à l'extrême fin du VIe ou au début du Ve s. avant J.-C. (Dedet, Py, 1973). Implantées dans une petite combe des Garrigues de la bordure occidentale de la Vaunage, au pied de l'habitat perché contemporain de la Font du Coucou, elles ont été découvertes en 1970 après défonçage d'une terre plantée en vigne. Ainsi, aucune n'était en place, mais les éléments composant les dépôts étaient restés groupés. Ces sépultures révèlent des pratiques différentes de celles observées aussi bien dans les tumulus à incinération qu'à la Font de la Vie. La tombe 3, retournée par la charrue mais la moins détruite des trois, montre le scénario suivant : quelques restes osseux brûlés du défunt ont été prélevés sur le bûcher et placés dans une urne non tournée faisant office d'ossuaire, en compagnie d'au moins quatorze bracelets-armilles en bronze qui, eux, ne portent pas trace de passage sur ce bûcher; l'ensemble a été déposé dans un loculus creusé en pleine terre où l'on a ajouté d'autres objets dépourvus de tout indice de feu secondaire (deux autres vases non tournés, une amphore étrusque - entière ou des fragments seulement - une fibule et quatre anneaux en bronze). Les tombes 1 et 4 apportent quelques confirmations et compléments d'information. Le mobilier métallique, six bracelets-armilles en bronze pour la tombe 4 et un couteau en fer pour la tombe 1 , n'est pas non plus passé sur le bûcher. En revanche, certains vases présentent des stigmates de feu secondaire : coupe attique à vernis noir de type $\mathrm{C}$ de Bloesch à lèvre concave et vase-support en céramique grise monochrome de la tombe 1. Comme la coupe a été retrouvée cassée mais presque complète, elle a dû être déposée entière dans la tombe après son passage sur le bûcher, et non brisée sur celui-ci comme à Font de la Vie. Le vase support est très incomplet et l'on ne peut savoir si cet état provient des dégâts ultérieurs causés à la sépulture (Dedet, Py, 1973).

La tombe de la Cougourlude à Lattes (Prades, 1979; Py, 1988, p. 109) est également datée de la fin du VI ${ }^{e}$ s. ou du début du $\mathrm{V}^{\mathrm{e}}$ s. par la présence d'une fibule annulaire en fer du type 4a de E. Cuadrado (Cuadrado, 1957, p. 14 15 et p. 45-46) ${ }^{7}$. Découverte à proximité immédiate d'un petit habitat du VIe s. avant J.-C. situé à $800 \mathrm{~m}$ au nord-est de la ville portuaire de Lattara, elle a été fortuitement mise au jour, et endommagée, par le creusement d'un fossé à la pelle mécanique. La connaissance que nous avons de sa forme comme de la composition du dépôt funéraire et des restes du défunt est extrêmement incomplète. Toutefois, les pratiques funéraires qu'elle suggère paraissent proches de celles dont témoignent les tombes de la Bergerie Hermet : cette sépulture semble en effet avoir été constituée d'un vase-ossuaire unique, en céramique non tournée, placé dans un loculus rempli, selon les termes de $\mathrm{H}$. Prades, « d'une terre cendreuse recouverte de galets "; l'ossuaire contenait «quelques ossements brûlés " et la fibule annulaire en fer, qui, vu son état de conservation jugé "stupéfiant », semble avoir échappé au bûcher (Prades, 1979).

Par contre, on ne possède aucun élément pour tenter d'identifier les usages en vigueur à Uzès lors de la constitution de la tombe de Préville ou du Château-Bérard, à la fin du IV $\mathrm{V}^{\mathrm{e}} \mathrm{s}$. ou dans la première moitié du siècle suivant

\footnotetext{
7. Cet auteur place ce type parmi les plus anciennes fibules annulaires, et de fait, on rencontre des objets semblables à celui de la Cougourlude dans la tombe Bonjoan 55 d'Ampurias, datée du premier quart du Ve $\mathbf{s}$. (Almagro, 1953, p. 193-196), ou dans la tombe 73 de Couffoulens II, dans l'Aude, datée de l'intervalle 520 - 480 (Passelac, Rancoule, Solier, 1981, p. 20 et 45). Un exemplaire de forme très proche, mais l'arc n'est pas élargi, provient de la tombe 232 de Saint-Julien à Pézenas, contemporaine de Couffoulens 73 (Giry, 1965, p. 211-212).
} 
(Saint Venant, 1897, p. 487-489). C'est une trouvaille ancienne, de 1887 , consécutive à un défonçage agricole. Seul le mobilier, aujourd'hui disparu, a retenu l'attention de J. de Saint Venant, qui en donne un inventaire dix ans plus tard. Mais, hormis pour une fibule en bronze à pied replié sur l'arc et à ornements de corail, qui seule a fait l'objet d'un dessin, l'identification précise des autres éléments, en particulier des récipients céramiques, est à présent problématique. La fibule ne paraît pas déformée par le feu. Donc, s'il s'agit bien d'une tombe à incinération (mais nous n'avons aucune indication sur les restes du mort et le sort que le cadavre a subi), cet objet d'habillement et de parure n'a pas séjourné sur le bûcher funèbre.

Pour le dernier quart du III et la première moitié du $\mathrm{II}^{\mathrm{e}}$ s. avant J.-C., nous disposons depuis peu d'un ensemble de sept sépultures fouillées dans la nécropolc du Sablas à Ambrussum à Villetelle, Hérault (Fiches dir., 1989, p. 27-40). Le niveau où se trouvent ces structures n'a été atteint pour le moment que dans des sondages d'extension limitée, menés sous un quartier d'habitat créé au I ${ }^{\text {er }}$ s. avant J.-C. : il pourra être exploré sur une surface plus importante après la fouille des couches qui le surmontent. Ces tombes laissent voir des éléments d'un rituel fort proches de ceux discernables à Font de la Vie. Elles consistent en petites fosses de 50 à $70 \mathrm{~cm}$ de diamètre pour 15 à $25 \mathrm{~cm}$ de profondeur, remplies par un sédiment prélevé en vrac sur l'ustrinum, et renfermant des os incinérés, des fragments de vases brisés sur le bûcher, quelques objets et offrandes alimentaires. Ce matériel d'accompagnement est toutefois beaucoup moins altéré par le feu que celui de Font de la Vie, témoignant sans doute d'une différence dans la disposition des objets sur le bûcher funèbre. Les stigmates du passage sur l'ustrinum apparaissent parfois - quelques traces de recuit sur des tessons, début de fusion pour un élément de ceinture en bronze - mais sont le plus souvent absentes, comme par exemple pour les deux perles en corail retrouvées parmi les ossements de la sépulture 31 . Ces tombes étaient dépourvues de toute couverture et parfois le dépôt funéraire déborde assez largement de la fosse. Elles ont été rapidement ensevelies sous les alluvions du Vidourle tout proche (Fiches dir., 1989, p. 27-40) ${ }^{8}$.

8. L'existence, sur le mobilier des tombes du Sablas, de traces de passage sur le bûcher funèbre nous a été précisée oralement par MM. JeanLuc Fiches et Michel Feugère; nous les en remercions.
Les quelques autres sépultures du $\mathrm{II}^{\mathrm{e}}$ s. avant J.-C. connues à ce jour renouent avec le rituel en vigueur à la Bergerie Hermet et à la Cougourlude et s'éloignent de celui d'Ambrussum et de la Font de la Vie : tombes des Colombes 3 et 4 à Beaucaire (respectivement premier quart et milieu du II ${ }^{\mathrm{c}}$ s.) (Dedet, Michelozzi, Py, 1974, p. 75-84), d'Atila à Sernhac (second quart du II ${ }^{\mathrm{e}}$ s.) (Py, 1983), de l'octroi de Beaucaire à Nîmes (milieu du $\mathrm{II}^{\mathrm{e}} \mathrm{s}$.) (Py, 1981b, p. 141-148) et de la gare de Nages (troisième quart du $\mathrm{II}^{\mathrm{e}} \mathrm{s}$.) (Py, 1980). Et de telles pratiques existent encore dans la même région dans les premières années du ${ }^{\text {er }}$ s. avant J.-C. comme l'atteste la sépulture du Mas de Jallon à Beaucaire (Garmy, Michelozzi, Py, 1981). Une partie des ossements du mort incinéré sur un ustrinum est prélevée et enfermée dans un vase-ossuaire : les Colombes 3, le Mas de Jallon sont les rares cas où les os ont été observés et conservés ${ }^{9}$. L'ossuaire est déposé dans un loculus creusé en pleine terre, environné de vases, d'objets métalliques, armes, parures ou ustensiles divers, et d'offrandes alimentaires ne portant pas les marques d'un passage sur le bûcher funéraire.

Durant le I ${ }^{\text {er }}$ s. avant J.-C., les sépultures en loculus persistent en milieu rural : Bergerie Hermet (tombe 2) à Calvisson (Dedet, Py, 1973, p. 51-52), Poligor à Combas, la gare à Nages, les Moureires à Saint-Côme-et-Maruéjols (Py, 1990, p. 317-324), Grand-Champ à Saint-Siffret (Dedet, 1983, p. 10), Saint-Laurent-des-Arbres et sans doute Sauveterre (Barruol, Sauzade, 1969), nécropole du Paradis à Aramon (Gallia, 37, 1979, p. 536), et au moins une des tombes du cimetière gallo-romain de la Javone à Rochefort du Gard (Gagnière, 1931). Mais en milieu urbain, elles sont progressivement remplacées par des tombes en coffre de dalles, à Nîmes (Camplanier 1 et 2, la Cigale, les Fontilles 1 et 2, le Mail) (Py, 1990, p. 317-324; Gallia Informations, 1992-1, p. 115) comme à Beaucaire (les Colombes 1, 2 et 5, les Marronniers 12, 17 et 18 et le Sizen 1) (Dedet, Michelozzi, Py, 1974; Dedet, Michelozzi et alii, 1978, p. 83-125). Dans tous les cas, les restes incinérés du défunt prennent place dans un vase-ossuaire, entouré d'un mobilier divers qui, sauf exception, comme les armes de la tombe de Camplanier I à Nîmes, dans le troisième quart du Ier s. avant J.-C., n'est pas brûlé (Py, 1990, p. 772 et 778).

\footnotetext{
9. Cependant, dans certains cas, comme à l'octroi de Beaucaire, à Nîmes, et à la gare de Nages, à Nages, les inventeurs, respectivement A. Vigne en 1891 et O. Rappaz en 1950, ont relevé l'absence de trouvaille d'ussements incinétés.
} 


\section{En guise d'épilogue}

Ia tombe fouillée en 1993 au licu-dit Font de la Vie est celle d'un individu adulte, très probablement un hommc, décédé au milieu du $\mathrm{V}^{\mathrm{e}}$ s. avant J.-C., dont le cadavre, accompagné d'un "riche " mobilier, a été brûlé sur un ustrinum. Elle comprend une fosse sépulcrale, où ont été jetés, en vrac, une petite partic du bûcher funèbre renfermant os incinérés ct quelques restes du matériel d'accompagnement fortement altéré par le feu, et un dépôt annexe probablement d'offrande. Son mode de couverturc, et éventuellement de signalisation, reste totalement inconnu du fait des conditions de gisement et de découverte. Les vestiges que laissent de telles pratiques peuvent disparaître rapidement sous l'effet des agents naturels, à la différence des tombes présentant une architecture importante et/ou un mobilier déposé intact. Leur mise au jour fortuite peut également passer très facilement inaperçue. Ainsi peut sans doute s'expliquer la grande indigence de la documentation en ce domaine, dans cette région, entre le début du Ve et le début du $\mathrm{II}^{\mathrm{e}} \mathrm{s}$. avant J-C.C., et seules des prospections systématiques et renouvelées sont susceptibles de remédier à cet état. Cette découverte constitue donc un jalon important pour discerner l'évolution des pratiques funéraires en Languedoc oriental à l'Âge du Fer. Plusieurs faits méritent d'être soulignés.

Tout d'abord, la variété des types de traitement du corps en vigueur aux VIII ${ }^{\mathrm{e}}-\mathrm{VII}^{\mathrm{c}}$ s. avant $\mathrm{J}$.-C. dans le complexe tumulaire, où défunts incinérés voisinent avec morts non brûlés, en dépôt secondaire ou primaire, disparaît au VIc s., cédant la place à l'unicité. Seule subsiste alors, dans les derniers tumulus, l'incinération, avec dépôt hors de tout contenant et souvent disséminés, de quelques os brûlés accompagnés d'objets qui ne sont pas passés par le bûcher.

À partir de la fin du VI' ou du début du Ve s., si l'incinération reste exclusive dans la région, durant tout le sccond Âge du Fer, du moins pour les individus décédés après la petite enfance ${ }^{10}$, deux grands types de pratiques apparaissent :

- le dépôt, dans un loculus creusé en pleine terre, d'un vase-ossuaire contenant quclques os humains prélevés sur le bûcher et parfois des objets de parure non brûlés,

10. En effet, la plupart des foctus, nouveau-nés, nourrissons et petits enfants connus sont alors inhumés dans les habitations ou à leurs abords, presque toujours sans trace perceptible de rituel funéraire, dans une petite fosse dépourvue de dispositif de couverturc ou de signalement (Dedet, Duday, Tillier, 1991). entouré du reste du mobilier d'accompagnement qui, sauf exception dans un cas pour des vases, n'a pas non plus séjourné sur le bûcher et est déposé complet. En témoignent la Bergerie Hermet 1, 3 et 4 et la Cougourlude vers 500 ou dans le premier quart du Ve s. avant J.-C., les Colombes 3 et 4, l'octroi de Beaucaire, la gare de Nages et la tombe d'Atila au II ${ }^{\mathrm{c}} \mathrm{s}$. avant J.-C., ainsi que le Mas de Jallon à la jointure des $\mathrm{II}^{\mathrm{c}}$ et $\mathrm{I}^{\mathrm{cr}} \mathrm{s}$. avant J.-C.;

- le dépôt en vrac, dans une petite fosse, d'une petite partie du bûcher, sédiment contenant des os incinérés non triés et des morceaux d'un mobilier brisé sur l'ustrinum. La Font de la Vie vers 450 et le Sablas à Ambmussum entre 225 et 175 rendent compte de tels usages, avec semble-t-il une différence dans la position du matériel accompagnant le mort sur le bûcher, celui de la Font de la Vie ayant été exposé à une chaleur beaucoup plus intense que celui du Sablas.

Ces formules alternèrent-elles dans lc temps, ou bien ont-elles eu cours simultanément et témoignent-elles d'une variabilité importante du rituel en usage au même moment et dans un même groupe humain, à l'instar du premier Âge du Fer? Une telle diversité du rituel funéraire dans un même groupe humain protohistorique à une époque donnée est un phénomène couramment constaté. On le retrouve dans nombre de sociétés historiques ou traditionnelles sub-actuelles. Pour celles-ci, les travaux des historiens et des ethnologues mettent en avant différentes raisons, le statut social du défunt, les modalités de sa mort, les causes de son décès et l'interprétation qu'en font les survivants (Dedet, 1992, p. 257263). En revanche on est beaucoup plus démuni pour tenter d'expliquer les raisons d'une évolution des pratiques funćraires en l'absence de toute trace permettant de discerner un changement ethnique. La contemporanéité entre Ambrussum ct les Colombes 3 au premier quart $\mathrm{du} \mathrm{II}^{\mathrm{e}} \mathrm{s}$. pourrait indiquer plutôt l'hypothèse de la variabilité, mais convenons que la documentation est trop clairseméc pour le moment pour permettre de conclure sur ce point.

Si la sépulture de la Font de la Vie est le premier exemple signalé en Ianguedoc oriental de dépôt en vrac de résidus de l'incinération dans une petite fosse avec mobilier funéraire fortement altéré par le feu, cette formule apparaît aussi, sensiblement à la même époque, dans la partie occidentale du Languedoc. Elle est ainsi attestée à partir de la fin du $\mathrm{VI}^{\mathrm{c}} \mathrm{s}$. et au début du siècle suivant dans plusieurs tombes du Grand-Bassin II à Mailhac (Aude) (tombes 2, 6, 12 et 13), de Couffoulens I (Aude) 
(tombes 13, 39, 40 et peut-être 27 et 41) et de Couffoulens II (tombes 46 à 50, 52 à 54, 56, 58 à 69, 71 et 72). Mais, à la différence de Font de la Vie, le loculus de ces sépultures est surcreusé, au fond, d'une excavation, souvenir de l'époque immédiatement antérieure, quand un vase-ossuaire était logé dans cette structure en creux (Rancoule, 1983, p. 43-45). Puis, à partir du début du $\mathrm{V}^{\mathrm{e}}$ s., le loculus n'est plus surcreusé, et la structure en creux du tombeau est en tous points semblable à celle de Font de la Vie : ainsi, dès le début du $V^{\mathrm{e}}$ s. à Couffoulens II (tombe 85) (Passelac, Rancoulc, Solicr, 1981, p. 26-27), et surtout, dans la seconde moitić de ce siècle, dans les tombes des Bosquets à Cesseras, Hérault (Rancoule, 1983), de Saint-Brès à Trausse-Minervois, Aude (Bernat, Rancoule, 1986, p. 110-111), du Traversant à Mailhac (Aude), et de la Martelle à Siran (Hérault) (Rancoule,
1989 , p. 45), ou encore, dans le dernier quart du $\mathrm{V}^{\mathrm{e}} \mathrm{s}$., dans certaines des plus anciennes tombes connues d'Ensérune (Schwaller, Marchand, 1993, p. 225). La tombe de Font de la Vie témoigne donc d'une évolution du rituel funéraire sur une aire géographique vaste, couvrant l'ensemble du Ianguedoc méditerranéen ${ }^{11}$.

Bernard DEDET

11. De teils usages n’ont pas été remarqués à ce jour en Provence, où la documentation concernant le milieu de l'Âge du Fer, pour ce domaine, est aussi rare qu'en I anguedoc oricntal. Sculs ceux que reflète la fosse funéraire $\mathrm{B}$ des Conques à Cucuron, Vaucluse, datée du $\mathrm{V}^{\mathrm{c}} \mathrm{s}$. avant J.-C., pourraient leur être rapprochés, mais os calcinés, bijoux brûlés et offrandes sont répartis en des tas bien distincts, et la fosse possède une bordure de blocs de grès (Dumoulin, 1962, p. 324-326). 


\section{Bibliographie}

Almagro M.

1953, Las necrópolis de Ampurias, I, Barcelone, 399 p. et XVIII pl.

ARNAL J., JEANJEAN L.

1936-1946, Deux stations préhistoriques de l'Hérault (canton de Castries), Bulletin de la Société d'Études Scientifiques de Nîmes, p. 101112.

BARRUOl G., SAUZADE G.

1969, Une tombe de guerrier à SaintLaurent-des-Arbres (Gard). Contribution à l'étude des sépultures du I ${ }^{\text {er }} \mathbf{s}$. av. J.-C. dans la basse vallée du Rhône, Revue d'Études Ligures, 35, p. 15-78.

BERnAT P., RANCOULE G.

1986, La nécropole protohistorique de Saint-Brès à Trausse-Minervois (Aude), observations sur quelques mobiliers de tombes de la fin du Premier Âge du fer de la vallée de l'Argent Double, Archéologie en Languedoc, 4, p. 103-111.

\section{BLOESCH H.}

1940, Formen attischer schalen von Exekias bis zum Ende des Strengen stils, Berne, 163 p. et $40 \mathrm{pl}$.

BOARDMAN J.

1975, Athenian Red Figure Vases. The Archaic Period, Londres, Thames and Hudson, $252 \mathrm{p}$.

1989, Athenian Red Figure Vases. The Classical Period, Londres, Thames and Hudson, 252 p.

Bonucci E., GRAZZiani G.

1975, Comparative

Thermogravimetric, X-ray

Diffraction and Electron

Microscope Investigations of Burnt Bones from Recent, Ancient and Prehistoric Age, Academia Nazionale dei Lincei, serie 8, 59, 5, p. 518-533.
Cuadrado Em.

1957, La fibula anular hispánica y sus problemas, Zephyrus, VIII, p. 5-76.

DEDET B.

1973, Les niveaux protohistoriques de l'oppidum de Vié-Cioutat à Mons, Monteils, Gard (fouilles 1966-1968), $R A N, \mathrm{VI}, \mathrm{p} .1-71$.

1978, Note sur la céramique tournée à gros dégraissant du Languedoc oriental, Figlina, 3, p. 24-42.

1983, Les origines protohistoriques d'Uzès, Rhodanie, 5, p. 7-11.

1992, Rites funéraires protohistoriques dans les Garrigues languedociennes, Paris, CNRS, 413 p. (24 ${ }^{\mathrm{c}}$ suppl. à la $R A N)$.

1995, Étrusques, Grecs et Indigènes dans les Garrigues du Languedoc oriental au Premier Âge du Fer : habitats et sépultures, in: Sur les pas des Grecs en Occident, Hommages à A. Nickels, p. 227-307 (Études Massaliètes, 4).

à paraître, Tombes et rites funéraires protohistoriques des Grands Causses.

Dedet B., DudAY H., Tillier A.-M. 1991, Les inhumations de fœetus, nouveau-nés et nourrissons dans les habitats protohistoriques du I anguedoc : l'exemple de Gailhan (Gard), Gallia, 48, p. 59-108.

Dedet B., Michelozzi A., Py M. 1974, La nécropole des Colombes à Beaucaire (Gard) (II $-^{\mathrm{e}} \mathrm{I}^{\mathrm{er}} \mathrm{s}$. av. J.-C.), RAN, VII, p. 59-118.

Dedet B., Michelozzi A., Py M., Raynaud C., Tendille C.

1978, Ugernum. Protohistoire de Beaucaire, Caveirac, 156 p. (ARALO, 6).
DEDET B., PY M.

1973, Les tombes protohistoriques de la Bergerie Hermet à Calvisson (Gard), Gallia, 31, p. 37-58.

Dumoulin A.

1962, I es fosses funéraires de Cucuron (Vaucluse), Gallia, XX, p. 323-333.

FÉDIÈRES A., GASCO Y.

1979 , Sauvetage du tumulus $\mathrm{n}^{\circ} 1$ des Tourelles à Buzignargues (Hérault), Bulletin de la Société Préhistorique Française, 76-6, p. 187-192.

FICHES J.-L. (dir.)

1989, L'oppidum d'Ambrussum et son territoire. Fouilles au quartier du Sablas (Villetelle, Hérault) : 1979-1985, Paris, CNRS, 286 p. (Monographie du CRA, 2).

GAGNIÈRE. S.

1931, Le cimetière gallo-romain de Rochefort-du-Gard, Rhodania, p. 207220.

GANTÈS L.-F.

1992, L'apport des fouilles récentes à l'étude quantitative de l'économie massaliète, in : Marseille grecque et la Gaule, p. 171-178 (Études Massaliètes, 3).

Garmy P., Michelozzi A., Py M. 1981, Une nouvelle sépulture protohistorique à Beaucaire (Gard) : la tombe du Mas de Jallon, RAN, XIV, p. 71-87.

GIRY J.

1965, La nécropole pré-romaine de Saint-Julien (commune de Pézenas, Hérault), Revue d'Études Ligures, 31, 1-2, p. 117-238.

GOUDINEAU Chr.

1975, Note sur le Sud-Est de la Gaule (Provence-Côte d'Azur), in : L'habitat et la nécropole à l'âge du Fer en 
Europe occidentale et centrale, Paris, Honoré Champion, p. 21-22.

GRUAT P., MARTY G.

1993, Découverte d'une tombe "aristocratique" du début du Premier Âge du Fer en Sévéraguais : le tumulus I de Roumagnac. Premiers résultats, in : Vivre en Rouergue, numéro spécial, Cahiers d'archéologie aveyronnaise, $7, \mathbf{p}$. 84-91.

\section{Hugues C.}

1960, Deux tumulus du Causse Noir et de la Can d'Artigues, Revue $d u$ Gévaudan, des Causses et des Cévennes, 6, p. 68-76.

JANNORAY J.

1955, Ensérune, contribution à l'étude des civilisations préromaines de la Gaule méridionale, Paris, De Boccard, 490 p.

JEHASSE J. et L.

1973, La nécropole pré-romaine d'Aléria, Paris, CNRS, 632 p. et 184 pl. (25 suppl. à Gallia).

LÉCRIVAIN Ch.

1896, Funus, Grèce, in : Daremberg Ch., Saglio Edm., Dictionnaire des Antiquilés grecques et romaines, 2, 2 partie (réed. 1969), p. 1367-1409.

LOUIS M., BRUGUIÈRE R.

1932, La Préhistoire de la vallée de la

Haute Bénovie, Mémoires de la Société Archéologique de Montpellier, X, 1, p. 137.

Passelac M., Rancoule G., Solier Y.

1981, La nécropole de "Las Peyros " à Couffoulens (Aude) : découverte d'un nouveau groupe de tombes, RAN, XIV, p. 1-70.

Prades $\mathrm{H}$.

1979, La fibule hispanique de la
Cougourlude, Joumal Communal de Lattes, 7, p. 20-21.

PY M.

1980, Une sépulture du II ${ }^{\mathrm{e}}$ s. av. J.-C. à Nages, Gard, RAN, XIII, p. 211-222.

1981 a, Les rites funéraires préromains de Nîmes et de sa région, Histoire et Archéologie, les dossiers, 55, p. 12-21.

1981 b, Recherches sur Nîmes préromaine, Paris, CNRS, 242 p. (41 ${ }^{\mathrm{e}}$ suppl. à Gallia).

1983, La tombe d'Atila (Sernhac, Gard, $\mathrm{II}^{\mathrm{e}} \mathrm{s}$. avant notre ère), RAN, XVI, p. 367-376.

1985, Les amphores étrusques de Gaule méridionale, Quaderni del Centro di Studio per l'Archeologia etrusco-italica, 9, p. 73-94.

1988, Sondages dans l'habitat antique de Lattes. Les fouilles d'Henri Prades et du groupe Archéologique Painlevé (1963-1985), Lattes, p. 65146 (Lattara, 1).

1990, Culture, économie et société protohistoriques dans la région nîmoise, Rome, 2 vol., 957 p., 302 ill. (Coll. EFR, 131).

PY M. dir.

1993, Dicocer ${ }^{1}$, Dictionnaire des céramiques antiques (VIIe s. av. n. è. - VIIe s. de n. è.) en Méditerranée nord-occidentale (Provence, Languedoc, Ampurdan), Lattes, 624 p. (Lattara, 6).

RANCOULE G.

1983, Tombes à incinération du début du Deuxième Âge du fer à Cesseras, Hérault, Bulletin de la Société d'Études Scientifiques de l'Aude, LXXXIII, p. 4150.
1989, Usages funéraires dans l'Aude au Premier Âge du Fer et au début du Second, Documents d'Archéologie Méridionale, 12, p. 41-49.

\section{ROUILLARD P.}

1992, La place de Marseille dans le commerce des vases attiques à figures rouges en Méditerranée occidentale (Ve-IV s. av. J.-C.), in : Marseille grecque et la Gaule, Lattes, p. 179-187 (Études Massaliètes, 3).

SAINT-VENANT J. de

1897, Les derniers arécomiques, traces de la civilisation celtique dans la région du Bas-Rhône, spécialement dans le Gard, Bulletin Archéologique du Comité des Travaux Historiques, p. 481-531.

\section{SANMARTÍ GREGo E.}

1976, Cerámicas de importación ática de El Puig de Benicarló (Castellón), Cuadernos de Prehistoria y Arqueología Castellonense, 3, p. 219-228.

SCHWALLER M., MARCHAND G.

1993, La phase tardive de la nécropole d'Ensérune (Hérault), in : Les Celtes en Normandie, Les rites funéraires en Gaule (IIT-Ir siècle avant J.-C.), Rennes, p. 225-229 (6 $6^{\mathrm{e}}$ suppl. à la $R A O)$.

\section{SPARKES B.-A., TALCOTT L.}

1970, Athenian Agora, XII, Black and Plain Pottery of the 6th, 5th and 4th Centuries B.C., 2 vol., Princeton.

Ugolini D., Olive C., MARChand G., COlumeau P.

1991, Béziers au Ve s. av. J.-C. Étude d'un cnscmblc dc mobilicr représentatif et essai de caractérisation du site, Documents d'Archéologie Méridionale, 14, p. 141-203. 